\title{
Envelope analysis of rotating machine vibrations in variable speed conditions: A comprehensive treatment
}

\author{
D. Abboud ${ }^{\mathrm{a}, \mathrm{b}, *}$, J. Antoni $^{\mathrm{a}}$, S. Sieg-Zieba ${ }^{\mathrm{b}}$, M. Eltabach ${ }^{\mathrm{b}}$ \\ a Laboratoire Vibrations Acoustique, Univ Lyon, INSA-Lyon, LVA EA677, F-69621 Villeurbanne, France \\ ${ }^{\mathrm{b}}$ Technical Center of Mechanical industries (CETIM), CS 80067, 60304 Senlis Cedex, France
}

\section{A R T I C L E I N F O}

\section{Article history:}

Received 26 August 2015

Received in revised form

4 May 2016

Accepted 20 June 2016

Available online 25 July 2016

\section{Keywords:}

Squared envelope spectrum

Envelope enhancement techniques

Variable speed conditions

Vibration analysis

Bearing diagnostic

\begin{abstract}
A B S T R A C T
Nowadays, the vibration analysis of rotating machine signals is a well-established methodology, rooted on powerful tools offered, in particular, by the theory of cyclostationary (CS) processes. Among them, the squared envelope spectrum (SES) is probably the most popular to detect random CS components which are typical symptoms, for instance, of rolling element bearing faults. Recent researches are shifted towards the extension of existing CS tools - originally devised in constant speed conditions - to the case of variable speed conditions. Many of these works combine the SES with computed order tracking after some preprocessing steps. The principal object of this paper is to organize these dispersed researches into a structured comprehensive framework. Three original features are furnished. First, a model of rotating machine signals is introduced which sheds light on the various components to be expected in the SES. Second, a critical comparison is made of three sophisticated methods, namely, the improved synchronous average, the cepstrum prewhitening, and the generalized synchronous average, used for suppressing the deterministic part. Also, a general envelope enhancement methodology which combines the latter two techniques with a time-domain filtering operation is revisited. All theoretical findings are experimentally validated on simulated and real-world vibration signals.
\end{abstract}

(c) 2016 Elsevier Ltd. All rights reserved.

\section{Introduction}

Envelope analysis has been recognized for long as a powerful technique for rolling element bearing (REB) diagnosis operating at constant speed. Typically, it consists of a bandpass filtering step around a frequency band wherein the impulsive excitation is amplified followed by a demodulation that extracts the signal envelope. The spectrum of the envelope known as the envelope spectrum - is expected to contain the desired diagnostic information, including the repetition rate of the fault and potential modulations [19]. At the time, the filtering and the demodulation operations were performed using analog techniques (e.g. [1]) with inherent limitations regarding the filter characteristics and the analog rectifier [11]. Thanks

\footnotetext{
Abbreviations: ; REB, rolling element bearings; SE, squared envelope; SES, squared envelope spectrum; CS, cyclostationary; SC, spectral correlation; VSC, variable speed conditions; ISA, improved synchronous average; CPW, cepstrum prewhitening; GSA, generalized synchronous average; SOI, source of interest; AT-CS, angle/time cyclostationary; BPOO, ball-pass-order on the outer-race

* Corresponding author at: Laboratoire Vibrations Acoustique, Univ Lyon, INSA-Lyon, LVA EA677, F-69621 Villeurbanne, France.

E-mail addresses: d-abboud@live.com, dany.abboud@insa-lyon.fr (D. Abboud), Jerome.antoni@insa-lyon.fr (J. Antoni), sophie.sieg-zieba@cetim.fr (S. Sieg-Zieba), mario.eltabach@cetim.fr (M. Eltabach).
} 


\begin{tabular}{|c|c|c|c|}
\hline \multicolumn{2}{|c|}{ Nomenclature } & \multirow{2}{*}{\multicolumn{2}{|c|}{$\begin{array}{c}\operatorname{cov}_{x, y}\left(t_{1}, t_{2} \mid \omega\right) \text { covariance of } x \text { and } y \text { conditioned to } \omega \\
\bar{\theta}\end{array}$}} \\
\hline$t$ & time variable & & \\
\hline$\theta$ & angle variable & $m_{Y}(\theta)$ & GSA trajectory tracked for a given speed pro- \\
\hline$\alpha$ & cyclic order variable & & file $\omega=\omega(\theta)$ \\
\hline$\omega$ & angular speed of the reference & $\delta \omega$ & speed resolution \\
\hline $\mathcal{F}\{*\}$ & Fourier transform & $\Phi$ & the angular sector spanned during the time \\
\hline $\mathbb{E}\{*\}$ & ensemble average & & record \\
\hline $\operatorname{SES}_{y}(\alpha)$ & squared envelope spectrum of signal $y$ & $\mathbb{R}$ & the set of real numbers \\
\hline $\mathrm{SE}_{y}(t)$ & squared envelope of signal $y$ & $\mathbb{Z}$ & the set of all integer numbers \\
\hline
\end{tabular}

to the advances of digital signal processing, considerable improvement has been made taking advantages of the Hilbert transform [3]. This latter returns the (complex) analytic signal whose modulus uniquely defines the envelope. In this context, it has been shown in Ref. [2] that it is preferable to use the squared envelope (SE) instead of the envelope inasmuch as the latter introduces extraneous components that appear as misleading peaks in the envelope spectrum. Since that time, the squared envelope spectrum (SES) has probably become the benchmark technique for bearing diagnostics, in particular due to its low computational cost [20].

During the last two decades, a wave of signal processing techniques rooted on the theory of cyclostationary (CS) processes [39] has appeared in multiple applications such as gears [13,40,42-44], REBs [11,45] and internal combustion engines [4648]. In particular, a new model of REB vibrations has been introduced in Refs. [2,11], providing an insightful understanding of the REB fault signature within the CS framework [9]. Accordingly, it has been shown that the mechanical signature generated by a faulty REB is random in nature and has symptomatic properties that can be detected by means of secondorder CS tools such as the SES, the spectral correlation, the spectral coherence, the cyclic modulation spectrum and others $[11,17,18,8,30]$. In this context, Ref. [11] has established the relationship between the spectral correlation and the SES. Since then, the SES has been considered as a (second-order) CS tool.

All the above techniques rely on the assumption of constant - or possibly fluctuating but in a stationary way - operating speed. Unfortunately, in some applications, the permanent acquisition of (quasi-) constant speed records is not available. A typical example is a wind turbine whose speed is mostly dependent on the random behavior of the wind. Evidently, the CS tools - including the SES - fail in describing such signals. Specifically, it has been shown in Refs. [27,29] that the vibrations emitted by rotating machines are likely to witness an interaction between time- and angle-dependent components. For instance, the REB fault signal can be viewed as a series of cyclic impacts locked to the shaft angle and exciting structural resonances $[4,27,33]$. Clearly, the positions of the impact excitations are dictated by the shaft angle while the resonance responses are governed by differential equations that impose time-invariant properties (e.g. natural frequencies and relaxation times). Therefore, efforts have been directed toward the extension of existing diagnostic tools in variable speed conditions (VSC). The recent advances in this framework can be classified into two directions.

1)In the first one, works have been oriented toward the coupling between the SES and computed order tracking to obtain an order domain representation. In order to deal with the non-consistency of the dynamical response and to eliminate potential interfering deterministic components, two general strategies have been adopted. The first strategy filters the time signal around a high-frequency resonance band (or simply high-pass filtering the signal) [23,37,38], while the second one eliminates the contribution of deterministic sources like gears with sophisticated "deterministic/random separation" tools and directly applies the SES on the residual signal $[20,28,26]$.

2)In the second direction, efforts have been oriented toward the generalization of existing CS tools. In particular, Urbanek et al. [35] proposed an angle-frequency distribution - namely the averaged instantaneous power spectrum - based on a time filtering step followed by an angle averaging operation of the squared output. A similar solution was proposed by Jabłoński et al. [5] who introduced the angular-temporal spectrum to jointly represent the angular and time properties of the signal. More interestingly, a prominent solution was proposed by D'Elia et al. [33] who explore the order-frequency approach. Their idea was to replace the frequency-frequency distribution by a frequency-order distribution that jointly describes the time-dynamics and the angle-periodicities of the signal. They proposed intuitive estimators for the spectral correlation and cyclic modulation spectrum which were coined as the $\alpha$-synchronized spectral correlation density and $\alpha$ synchronized cyclic modulation spectrum, respectively. Later on, a fast version of the former estimator, called "speed correlation", was proposed in Ref. [34] wherein the speed transform was used in the algorithm. Recently, Refs. [16,29] set up the foundations of angle/time cyclostationarity which extend the CS framework to enfold speed-varying signals. These references equally provide (i) a rigorous definition of the order-frequency spectral correlation together with its normalized form (namely the order-frequency spectral coherence), as well as a consistent Welch-based estimator.

This paper is particularly concerned with the first direction and tries to organize existing works into a structured comprehensive framework. It is intended to serve as a guideline on how to exploit the SES in rotating machine diagnosis and to optimize it under given operating conditions. Overall, this paper also brings a number of original results that provide a more comprehensive view of some previously published materials. 
This paper is organized as follows: Section 2 introduces a phenomenological signal model that describes the different components to be expected in the SES. This model will serve to formalize and illustrate the basic problem in VSC. Section 3 examines existing envelope enhancement strategies. In particular, it evaluates and compares three sophisticated deterministic random separation techniques, namely, the improved synchronous average (ISA) [21,23], the cepstrum prewhitening (CPW) [24,25] and the generalized synchronous average (GSA) [26]. Also, it addresses a general strategy to enhance a random source of interest - e.g. a REB fault component - and to enhance its emergence in the SES. All the theoretical findings and the qualitative comparisons are validated on simulated signals in Section 5 and on real vibration signals including various case studies in Section 6. Eventually, the paper is sealed with a general conclusion in Section 7.

\section{Problem formulation}

The object of this section is to investigate the sources of interference which are likely to enter the SES of a vibration signal. A phenomenological model of the different possible contributions is first provided in VSC. Based on this model, the SES of a vibration signal is analytically investigated and illustrated.

\subsection{Preliminaries}

According to Refs. [12-14], vibration signals generated by rotating machinery can be decomposed into three contributions of different statistical natures as

$$
x(t)=d(t)+r(t)+b(t), x(t)=d(t)+r(t)+b(t),
$$

where $t$ stands for the time variable, $d(t)$ for the deterministic contribution, $r(t)$ for the (random) cyclostationary contribution and $b(t)$ for the stationary contribution that is neither periodic nor CS and that will be referred to subsequently as background noise. The deterministic part is typically related to deterministic excitations such as gear vibrations, misalignments, unbalances, blade rotations, reciprocating forces, etc. In (quasi-) constant speed conditions, the deterministic part is intrinsically periodic in the angular domain; thus it exhibits a discrete (order-) spectrum [7,10]. The CS contribution is generated by random and repetitive excitations such as wear, friction forces, impacting forces, fluid motions, turbulence, combustion forces, etc. [10]. In this context, CS components are characterized by a periodic mean instantaneous power, thus exhibiting a discrete SES $[2,8,11]$. Last, the background noise is typically generated by non-cyclostationary sources such as environmental noise, sensor measurement errors, etc.

In VSC, rotating machine vibrations undergo significant changes in their properties. In particular, the deterministic contribution $d(t)$ loses its (angle-) periodicity, the CS contribution $r(t$ ) becomes cyclo-non-stationary (CNS) [15,16], and the background noise $b(t)$ becomes nonstationary (but also non-CNS). When analyzing rotating machine vibrations, the goal is typically to detect a particular vibratory component generated by a source of interest (SOI). The present paper is particularly concerned with the detection of a cyclo-non-stationary SOI using envelope analysis. In the case when the component produced by the SOI is of deterministic nature, other techniques must be implemented to detect it. Let $s(t)$ be the CNS component generated by the SOI and $q_{m}(t)$ be the $m$ th contribution generated by other interfering sources, so that:

$$
r(t)=s(t)+\sum_{m=1}^{M} q_{m}(t)
$$

where $M$ stands for the number of interfering CNS sources. Therefore, by separating the vibrational component into signal and noise, Eq. (1) becomes

$$
x(t)=s(t)+n(t)
$$

where

$$
n(t)=d(t)+\sum_{m=1}^{M} q_{m}(t)+b(t)
$$

stands for the noise which comprises the sum of the deterministic interferences $d(t)$, CNS interferences $\sum_{m=1}^{M} q_{m}(t)$ and background noise $b(t)$.

The common practice in the literature consists of combining the SES with computed order tracking to obtain an orderdomain representation [19,20,28,37,38]. This follows from the fact that the periodicity of the statistics of the CNS component may be preserved to some extent in the angular domain since machine kinematics is inherently dependent on angle. In this regard, two practical difficulties are expected. The first one is related to the deterministic interference in the SES, while the second one is related to the CNS interferences. In both cases, the signature of the SOI is polluted in the SES. The present section aims to model and illustrate this issue. For this purpose, a phenomenological model of the deterministic, CNS and background noise contributions in VSCs is provided in Section 2.2 in order to understand the structure of these components. 
This model will also serve to analytically investigate their contributions in the SES in Section 2.3.

\subsection{Generative model for vibration signals in variable speed conditions}

This subsection models the deterministic contribution, the CNS contribution and the background noise. Note that the deterministic contribution model presented in Section 2.2.1 is borrowed from a previous paper [26], yet the interpretations and illustrations are new. The material in Sections 2.2.2 and 2.2.3 is fully original.

\subsubsection{Modeling the deterministic part}

In nonstationary conditions, the deterministic component is subjected to significant amplitude modulation and phase blur. These are basically induced by the variation of the machine power intake [26,49-52,55] and the effect of time-invariant transfer functions from the excitation sources to the sensor [22,23]. The first effect results in amplitude modulation, whereas the second one also induces phase blur. Provided that the speed profile is sufficiently smooth, a realistic modeling of the deterministic component is provided in Ref. [26] through a Fourier decomposition whereof the coefficients have an explicit dependence on the speed, viz

$$
d(t)=\sum_{\lambda_{i} \in \Lambda} d^{\lambda_{i}}(\omega(t)) e^{j \lambda_{i} \theta(t)},
$$

where $\theta$ denotes the rotational angle (in [rad]) of the machine (typically the reference angular period equals $2 \pi$ radians), $\omega(t)$ denotes the instantaneous speed (in [ $\mathrm{rad} / \mathrm{s}]$ ) of the machine, $\lambda$ is a dimensionless index that denotes the machine cyclic orders (in events per revolution [evt/rev]), $\Lambda=\left\{\lambda_{i} \in \mathbb{R} / i=1,2 \ldots\right\}$ is the set of the cyclic orders associated with deterministic cyclic sources ( $\mathbb{R}$ is the real set), and $d^{\lambda_{i}}(\omega)$ are deterministic, complex, speed-dependent Fourier coefficients. In words, the deterministic component can be seen as a sum of angle periodic exponentials, $e^{j \lambda_{i} \theta(t)}$, modulated with speed-varying complex envelopes $d^{\lambda_{i}}(\omega(t))$. The former synchronize the waveform with the reference shaft frequency, while the latter account for the variation of the amplitude and phase induced by the transfer function of the machine.

The order-domain counterpart is simply obtained by applying the variable change $t=t(\theta)$ followed by a Fourier transform with respect to $\theta$, i.e.

$$
D(\alpha) \stackrel{\text { def }}{=} \mathcal{F}_{\theta \rightarrow \alpha}\{d(t(\theta))\}=\sum_{\lambda_{i} \in \Lambda} D^{\lambda_{i}}\left(\alpha-\lambda_{i}\right),
$$

where $\alpha$ stands for cyclic order variable, a quantity without unit that counts the number of events occurring per rotation of the reference shaft (the reference order is 1$), \mathcal{F}_{\theta \rightarrow \alpha}\{*\}=\lim _{\Phi \rightarrow \infty}(1 / \Phi) \int_{-\Phi / 2}^{+\Phi / 2}\{*\} e^{j \alpha \theta} d \theta$ stands for the Fourier transform $(\Phi$ is an angular sector) , $D^{\lambda_{i}}(\alpha)=\mathcal{F}_{\theta \rightarrow \alpha}\left\{d^{\lambda_{i}}(\tilde{\omega}(\theta))\right\}$ is the Fourier transform of $d^{\lambda_{i}}(\tilde{\omega}(\theta))$ having an order-domain bandwidth $B_{w}^{\lambda_{i}}$ and $\tilde{\omega}(\theta)=\omega(t(\theta))$ is the speed profile in the angular domain. It is worth noting that $B_{w}^{\lambda_{i}}$ depends on (i) the variability of the coefficient $d^{\lambda_{i}}(\omega)$ with respect to the speed (i.e. higher partial derivatives of $d^{\lambda_{i}}(\omega)$ with respect to $\omega$ ) and (ii) the variability of the speed profile itself (i.e. higher derivatives of $\tilde{\omega}(\theta)$ with respect to $\theta$ ). The more important these variabilities are, the wider the induced bandwidths are.

In a general way, these bandwidths are much narrower than the reference order (i.e. $\left.B_{w}^{\lambda_{i}} \ll 1\right)$ so that $D^{\lambda_{i}}\left(\alpha-\lambda_{i}\right)$ and $D^{\lambda_{i-1}}\left(\alpha-\lambda_{i-1}\right)$ do not overlap over the $\alpha$-axis for all $i=1,2 \ldots$ card $\{\Lambda\}$. As a consequence, the order spectrum also seems discrete along the $\alpha$-axis, yet undergoing an energy leakage of width $B_{w}^{\lambda_{i}}$ rather than being perfectly localized at the synchronous orders (i.e. $\lambda_{i} \in \Lambda$ ). In the particular case of constant speed the order spectrum is perfectly discrete as the bandwidths $B_{w}^{\lambda_{i}}$ tend to zero. The effect of the speed on the deterministic component is illustrated in Fig. 1.

\subsubsection{Modeling the cyclo-non-stationary part}

In the last few years, the term "cyclo-non-stationary" has appeared in the field of rotating machine to describe the signals produced when the operating conditions are strongly NS. Similarly to CS signals, they can be produced by gears [32,5355,66], REBs [33,56,57], internal combustion engines [15] and certainly other systems. A tentative definition of CNS signals was provided in [16] through a Fourier series model with mutually nonstationary stochastic coefficients, $y^{\gamma_{i}}(t)$,

$$
y(t)=\sum_{\gamma_{i} \in \Gamma_{y}} y^{\gamma_{i}}(t) e^{j \gamma_{i} \theta(t)}
$$

where $\Gamma_{y}=\left\{\gamma_{i} \in \mathbb{R} / i=1,2 \ldots\right\}$ is the associated set of the cyclic orders. As admitted by the authors, the time functions entering this definition are not unique: as a consequence it comprises all nonstationary signals since modulations can either be coded by the complex exponentials or by their nonstationary coefficients. It is clear that some constraint must govern the nonstationarity of these coefficients. Inspired from the deterministic model provided in Ref. [26] and reviewed in Section 2.2.1, the nonstationarity of the coefficients will be modeled through an explicit dependence on the nonstationary speed profile. Precisely, a general model of CNS signal can be expressed by the following decomposition: 

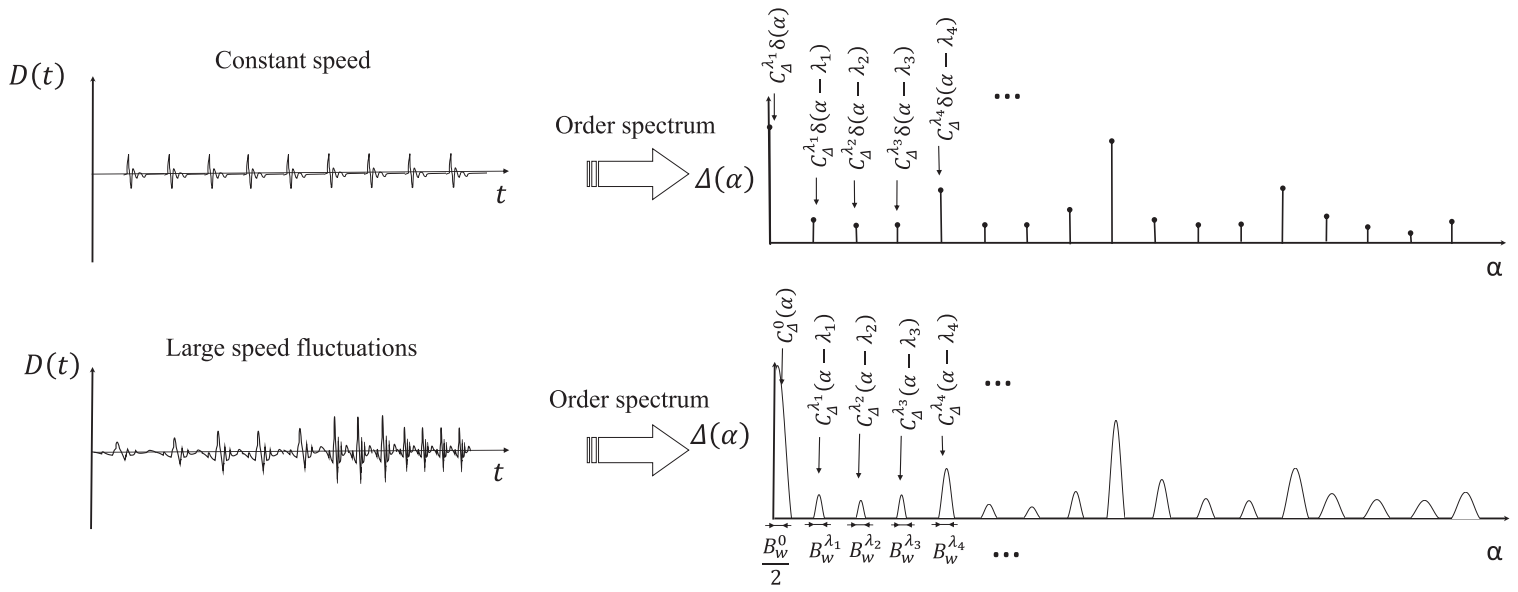

Fig. 1. Academic example illustrating the effect of the speed on the deterministic component in rotating machine vibrations.

$$
y(t)=\sum_{\gamma_{i} \in \Gamma_{y}} y^{\gamma_{i}}(t, \omega(t)) e^{\mathrm{j} \gamma_{i} \theta(t)},
$$

where $c_{Y}^{\gamma}(t, \omega)$ are mutually stationary stochastic process whose joint probability density function is conditioned on $\omega$ i.e. $\operatorname{cov}_{y^{\gamma} 1, y^{\gamma} 2}\left(t_{1}, t_{2} \mid \omega\right)=\sigma_{y^{\gamma} 1, y^{\gamma / 2}}^{2}\left(t_{1}-t_{2}, \omega\right)$. For a constant speed, the coefficients are stationary and Eq. (8) boils down to the wellknown Fourier decomposition of CS processes [10]. Conversely, these coefficients become nonstationary when the speed varies. Loosely speaking, the long-term changes in the CNS signal are expressed by the explicit dependence of the Fourier coefficients on the speed, while hidden periodicities are still conserved in the form of periodic modulations carried by the complex exponentials. According to that, the CNS components emitted by the SOI and by the $m$ th interfering source read:

$$
\left\{\begin{array}{c}
s(t)=\sum_{\gamma_{i}^{(s)} \in I_{S}} s_{i}^{\gamma_{i}^{(s)}}(t, \omega(t)) e^{j \gamma_{i}^{(s)} \theta(t)} \\
q_{m}(t)=\sum_{\gamma_{i}^{(m)} \in I_{m}} q_{m}^{\gamma_{i}^{(m)}}(t, \omega(t)) e^{j \gamma_{i}^{(m)} \theta(t)},
\end{array}\right.
$$

where $\Gamma_{s}=\left\{\gamma_{i}^{(s)} \in \mathbb{R} / i=1,2 \ldots\right\}$ and $\Gamma_{m}=\left\{\gamma_{i}^{(m)} \in \mathbb{R} / i=1,2 \ldots\right\}$ stand for the set of the cyclic orders associated with the SOI and the $m$ th source, respectively, and $s^{\gamma_{i}}(t, \omega)$ and $q_{m}^{\gamma_{i}}(t, \omega)$ are similar to the Fourier coefficients $y^{\gamma_{i}}(t, \omega)$ encountered in Eq. (8).

\subsubsection{Modeling the background noise}

As previously pointed out, the background noise is a random contribution generated by other sources not accounted for in the model and assumed to be stationary for constant operating speed. Therefore, no periodic modulations associated with non-zero cyclic orders are expected. Moreover, similarly to the CNS case, this contribution is likely to depend on the operating speed. Inspired by the CNS model introduced in Section 2.2.1, a model of the background noise can be obtained by simply replacing the cyclic order set $\Gamma$ in Eq. (8) with the singleton $\{0\}$, giving

$$
b(t)=b^{0}(t, \omega(t)),
$$

where $b^{0}(t, \omega)$ is a stationary stochastic process whose joint probability density function is conditioned on $\omega$. Having modeled the deterministic contribution, the CNS contribution and the background noise, it remains to analytically investigate their contributions in the SES. The next subsection addresses this issue.

\subsection{Interference of sources}

The aim of this section is to analytically investigate the limitations of the SES technique in analyzing the CNS component emitted by an arbitrary SOI. Based on the model proposed in Section 2.2, the contributions of the signal components in the SES are calculated and illustrated. Detailed calculations are provided in Appendix A. The SES is defined in the order domain as:

$$
\operatorname{SES}_{x}(\alpha) \stackrel{\text { def }}{=} \mathcal{F}_{\theta \rightarrow \alpha}\left\{S E_{x}(\mathrm{t}(\theta))\right\},
$$

where $S E_{x}(\mathrm{t})=\mathbb{E}\left\{|x(t)|^{2}\right\}$ stands for the SE of $x(t)$ and the Fourier transform is to be taken in the sense of distributions. Assuming that the signal components are uncorrelated and according to Eq. (3), the SES of the vibration signal equals the 
sum of the SES of each component on the average, i.e.

$$
\operatorname{SES}_{x}(\alpha)=\operatorname{SES}_{S}(\alpha)+\operatorname{SES}_{n}(\alpha) .
$$

The SES of the SOI takes the particular form

$$
\operatorname{SES}_{S}(\alpha)=\sum_{\gamma_{i}^{(S)} \in \Gamma_{S}} \operatorname{SES}_{S}^{\gamma_{i}^{(S)}}\left(\alpha-\gamma_{i}^{(s)}\right)
$$

where quantities $S E S_{S}^{\gamma_{i}^{(s)}}$ have the same interpretation as the functions $D^{\lambda_{i}}(\alpha)$ encountered in Eq. (3). In other words, they evidence a discrete distribution at the synchronous orders with possible energy leakage. Nevertheless, they still provide a symptomatic and informative distribution of the cyclic content. Likewise, the SES of the noise equals the sum of the SES of its components, i.e.

$$
\operatorname{SES}_{n}(\alpha)=\operatorname{SES}_{d}(\alpha)+\sum_{m=1}^{M} \operatorname{SES}_{q_{m}}(\alpha)+\operatorname{SES}_{b}(\alpha),
$$

with

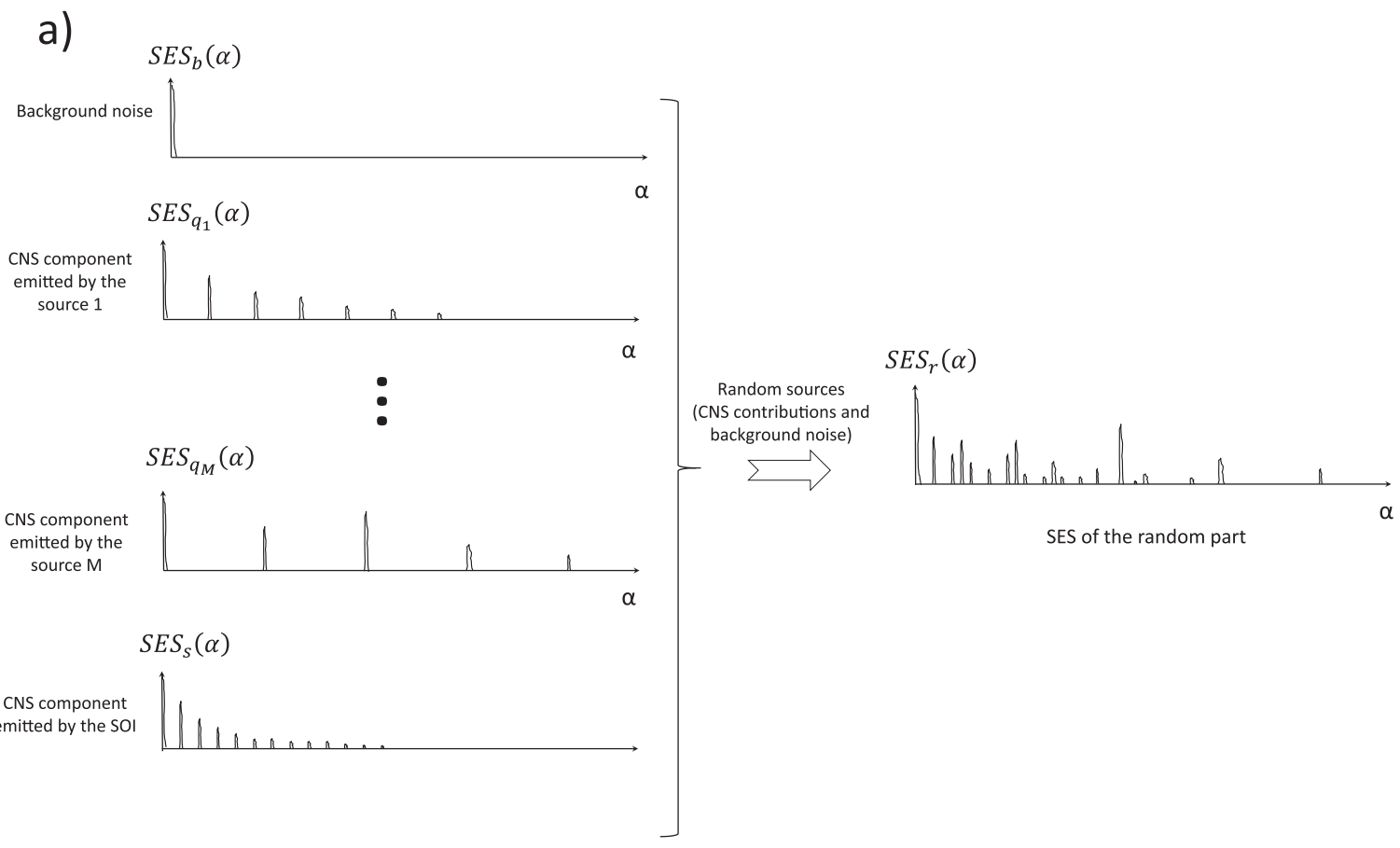

b)
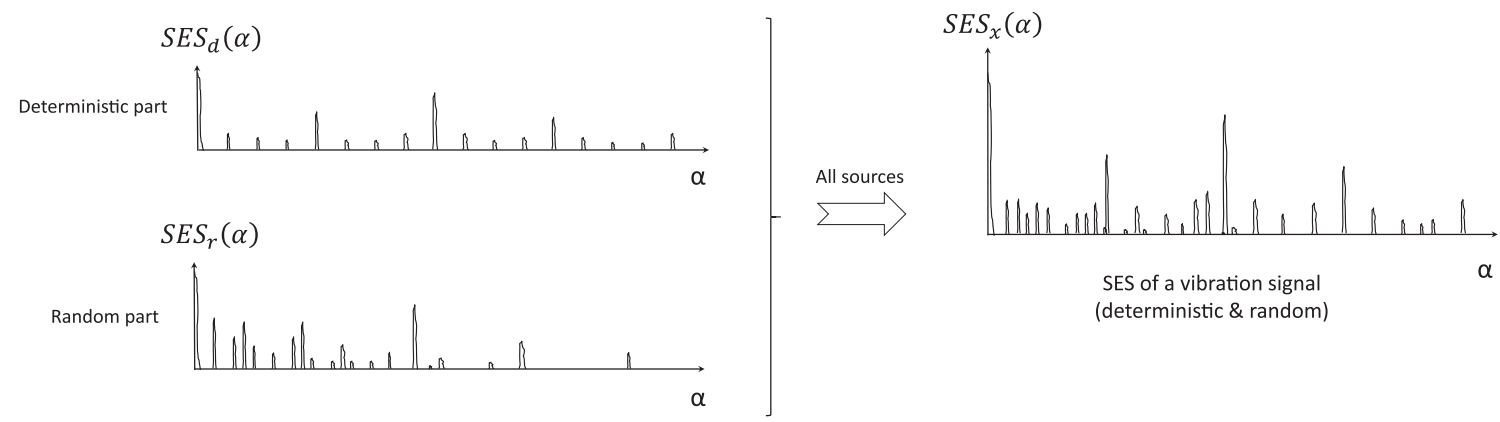

Fig. 2. Academic example illustrating the interference of different sources in the SES of a vibration signal: (a) interference among random sources (CNS components and background noise) and (b) interference between deterministic and random sources. 


$$
\left\{\begin{array}{c}
\operatorname{SES}_{d}(\alpha)=\sum_{\lambda_{i} \in \Lambda} \operatorname{SES}_{d}^{\lambda_{i}}\left(\alpha-\lambda_{i}\right) \\
\operatorname{SES}_{q_{m}}(\alpha)=\sum_{\gamma_{i}^{(m)} \in I_{m}} \operatorname{SES}_{q_{m}}^{\gamma_{m}^{(m)}}\left(\alpha-\gamma_{i}^{(m)}\right) \\
\operatorname{SES}_{\mathrm{b}}(\alpha)=\operatorname{SES}_{b}^{0}(\alpha),
\end{array}\right.
$$

where $S E S_{d}^{\lambda_{i}}(\alpha-\lambda), S_{q_{m}}^{\gamma_{i}^{(m)}}(\alpha-\gamma)$ and $S E S_{b}^{0}(\alpha)$ again have the same interpretation as the functions $D^{\lambda_{i}}(\alpha)$ encountered in Eq. (6). It is obvious that the SES of a vibration signal is composed of numerous interfering sources in general. The contribution of the background noise does not have a significant impact in the SES as its corresponding distribution is localized in the vicinity of zero (see the third row in Eq. (15)). However, the presence of other components in the SES - either of deterministic (first component in Eq. (14)) or CNS nature (second component in Eq. (14)) - will certainly complicate the analysis of the SES of the SOI. This will compromise the efficiency of the SES technique in the general setting. An illustration of this effect is provided in Fig. 2. Despite its presence in the SES of the random part, the contribution of the SOI component is hardly noticeable (see Fig. 2(a)): it is drowned among other CNS components from other sources. The problem is aggravated in the SES of the total vibration signal where the deterministic component also contributes to the SES (see Fig. 2 (b)). Another aspect of the CS source interference problem is the resulting bias in the SES estimator which jeopardizes hypothesis tests (generally used to detect second-order components) [17]. This problem has been firstly identified in Ref. [65] by analytically analyzing the error introduced by CS1 and CS2 sources. Recently, a new unbiased indicator, namely the Log-envelope spectrum, was proposed in Ref. [64] to deal with multiple CS2 sources.

In any case, the identified issues require the preprocessing of the signal before the application of the SES. The next section addresses this issue.

\section{Envelope enhancement strategies}

A current practice in VSC is to apply the (order-domain) SES over the whole demodulation band of either the whitened [25] or the residual [22,26] signal. Undoubtedly, the complete elimination of the deterministic component cleans the SES from its spurious contributions. For this purpose, Section 3.1 addresses the elimination of the deterministic component in VSC through the comparison of three sophisticated techniques previously conceived for this purpose.

Removing the deterministic part is a crucial but not sufficient step to exhibit the SOI signature in the SES: the SOI signature may still be masked by other CNS components (see Fig. 2(b)) [27]. For this purpose, Section 3.2 revisits a general procedure to enhance the SOI component in the signal and, consequently, enhances its emergence in the SES.

\subsection{Removing the deterministic part}

As indicated in subsection 2.2.1, the deterministic component undergoes significant speed dependent changes that can jeopardize its (angle-) periodicity. This necessarily invalidates the working assumption of conventional deterministic/random separation techniques despite being applied in the angular domain. Lately, some sophisticated preprocessing techniques have been proposed to deal with this issue. This section reviews and compares three of them, namely, the ISA, the CPW and the GSA. Specifically, the fundamentals of these techniques are summarized and qualitative evaluations of their performances are given.

\subsubsection{The improved synchronous average (ISA)}

After inspecting the phase blur resulting from the transmission path effect, Stander and Heyns [22] proposed an enhanced version of the SA - coined "phase domain averaging", being based on a phase domain representation. The basic idea is to order track the signal with respect to its own phase instead of that of the tachometer. The signal phase is obtained by Hilbert demodulation of a high-energy harmonic (such as a meshing order) of the angular signal. Cycles are then separately order tracked (for the second time) according to the estimated phase and then averaged together.

Later, a simpler variant was provided in Ref. [21] - coined the "improved synchronous average" (ISA) - where the authors proposed to resample the signal with a virtual tachometer signal synthesized via the demodulated phase. Subsequently, the technique was used in Ref. [23] where the authors analytically tried to identify the optimal (order-domain) demodulation band and proposed it as a discrete-random separation technique in VSC. The ISA technique consists of the following steps:

1) Order track the time vibration signal using the tachometer signal.

2) Band-pass filter the angular signal around a high-energy harmonic (the energy leakage must be included in the filter bandwidth).

3) Compute the analytic signal.

4) Construct a second virtual tachometer signal based on the phase of the analytic signal. 
5) Again, order track the angular signal using the virtual tachometer. The obtained signal is represented in the phase domain.

6) Apply the SA to obtain the ISA.

The flowchart of the ISA is shown in Fig. 3.

In the following, the ISA is investigated on the deterministic model provided in Eq. (5). Let us restate the model after expanding its Fourier coefficients:

$$
\tilde{d}(\theta)=d(t(\theta))=\sum_{\lambda_{i} \in \Lambda} A_{d}^{\lambda_{i}}(\tilde{\omega}(\theta)) \mathrm{e}^{\mathrm{j}\left(\lambda_{i} \theta+\Psi_{d}^{\lambda_{i}}(\tilde{\omega}(\theta))\right)},
$$

with $A_{d}^{\lambda_{i}}(\omega) e^{j\left(\psi_{d}^{\lambda_{i}}(\omega)\right)}=d^{\lambda_{i}}(\omega)$. Let $\lambda_{m}$ be the order of a high-energy harmonic (used for the phase demodulation), according to the first 3 steps of the ISA technique, the analytic signal of the filtered signal writes

$$
\tilde{d}_{f}(\theta)=d^{\lambda_{m}}(\tilde{\omega}(\theta)) e^{j \lambda_{m} \theta}=A_{d}^{\lambda_{m}}(\tilde{\omega}(\theta)) e^{j\left(\lambda_{m} \theta+\Psi_{d}^{\lambda}(\tilde{\omega}(\theta))\right)},
$$
i.e.

The reference signal phase is then obtaining by dividing the demodulated phase, $\lambda_{m} \theta+\Psi_{d}^{\lambda_{m}}(\tilde{\omega}(\theta))$, by the harmonic order,

$$
\theta^{\prime}=\theta+\frac{1}{\lambda_{m}} \Psi_{d}^{\lambda_{m}}(\tilde{\omega}(\theta))
$$

The second order tracking operation (step 5) is equivalent to representing the signal with respect to $\theta^{\prime}$. It can be shown that the phase blur of the signal can be completely compensated,

$$
\tilde{d}^{\prime}\left(\theta^{\prime}\right)=\sum_{\lambda_{i} \in \Lambda} A_{d}^{\lambda_{i}}\left(\tilde{\omega}^{\prime}\left(\theta^{\prime}\right)\right) \mathrm{e}^{\mathrm{j} \lambda_{i} \theta^{\prime}},
$$

where $\tilde{\omega}^{\prime}\left(\theta^{\prime}\right)$ is the speed profile in the phase domain, only if

$$
\lambda_{i} \Psi_{d}^{\lambda_{m}}(\omega)=\lambda_{m} \Psi_{d}^{\lambda_{i}}(\omega)
$$

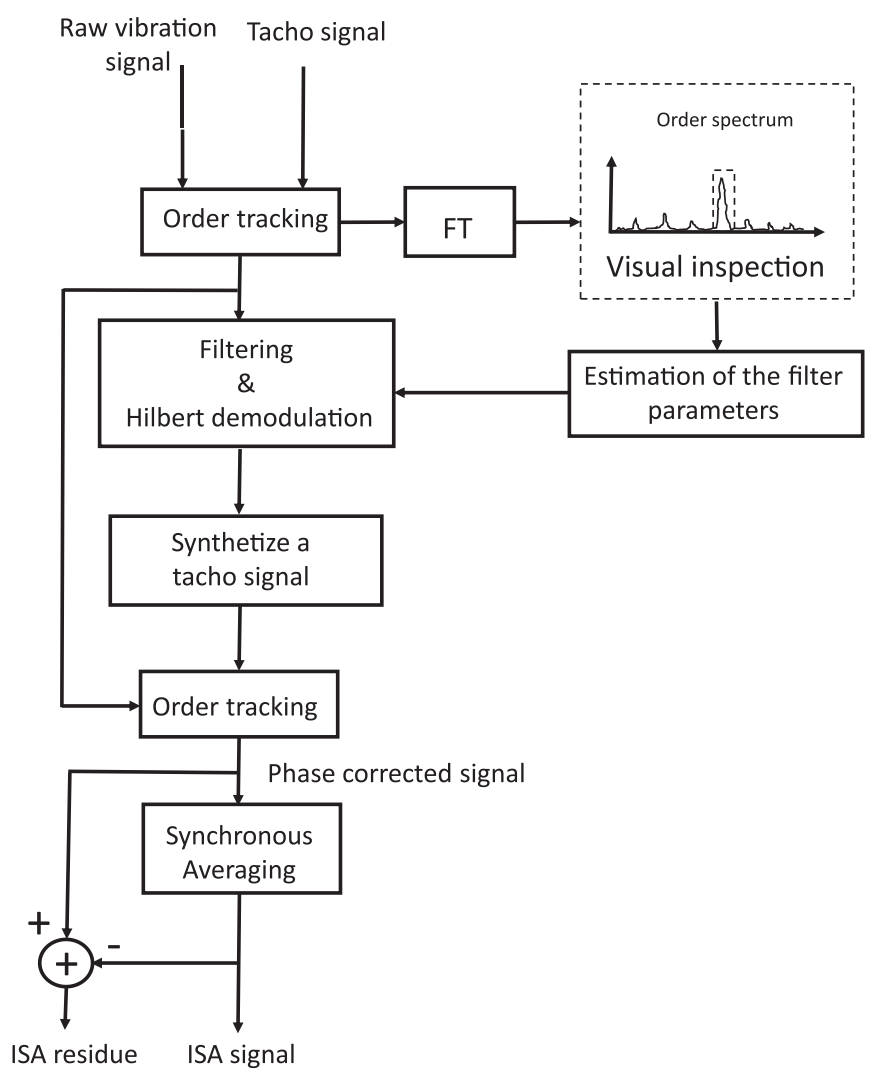

Fig. 3. Flowchart of the ISA. 
But, this equality implicitly assumes that the phase blurs associated with all the harmonics are linear between each other's. This assumption, however, is not always accurate; particularly when the spectral content of the harmonic coincides with a resonance. For instance, if one extracts the phase information from a meshing orders at a critical speed (i.e. in the vicinity of a structural mode); the latter is more subjected to phase blur than elsewhere [23,58]. As a consequence, only neighboring orders will be corrected when the speed variation is high, rather than distant ones. Note that, regardless of its capacity of correcting the signal phase, this technique ignores the long-term energy modulations in the signal. Therefore, the ISA cannot accommodate the amplitude variations and returns an averaged result. Despite its relevance in some applications, the ISA may suffer from serious issues in large speed variations; the reason it will not be considered further. This claim is experimentally evidenced in Sections 4 and 5 on simulated and real vibration signals, respectively.

\subsubsection{The cepstrum prewhitening (CPW)}

With the aim of enhancing fault detection in REBs, the authors of Refs. [20,24] introduced a powerful prewhitening technique - called cepstrum prewhitening (CPW) - based on cepstral analysis. This technique enjoys a remarkable capability of blindly eliminating the deterministic content in the signal and of returning a pure random whitened signal. Recently, Borghesani et al. [25] have applied the technique in VSC after order tracking the signal. Precisely, the CPW technique consists of the following steps:

1) Order track the time signal $\tilde{y}(\theta)=y(t(\theta))$.

2) Compute the Fourier transform,

$$
Y(\alpha)=\mathcal{F}_{\theta \rightarrow \alpha}\{\tilde{y}(\theta)\}=A_{Y}(\alpha) \cdot \mathrm{e}^{\mathrm{j} \Psi_{Y}(\alpha)}
$$

and save the phase $\Psi_{Y}(\alpha)$.

3) Compute the real cepstrum defined as:

$$
y_{r c}(\theta)=\mathcal{F}_{\alpha \rightarrow \theta}^{-1}\{\log (|Y(\alpha)|)\}
$$

4) Set to zero the real cepstrum except for the zero frequency.

$$
\left\{\begin{array}{c}
y_{w}(\theta)=y_{r c}(\theta) \quad \text { for } \theta=0 \\
y_{w}(\theta)=0 \quad \text { for } \theta \neq 0
\end{array}\right.
$$

5) Back to the order domain:

$$
Y_{w}(\alpha)=\exp \left(\mathcal{F}_{\theta \rightarrow \alpha}\{\tilde{y}(\theta)\}\right)
$$

6) Combine $Y_{w}(\alpha)$ with the original phase and back to the angle domain

$$
y_{C P W}(\theta)=\mathcal{F}_{\alpha \rightarrow \theta}^{-1}\left\{Y_{w}(\alpha) \cdot \mathrm{e}^{\mathrm{j} \Psi_{Y}(\alpha)}\right\}
$$

The flowchart of the CPW is shown in Fig. 4(a). It is worth noting that the previously described technique considers the theoretical derivation of the CPW. Alternatively, this latter can be computed in a simpler way as:

$$
y_{C P W}(\theta)=\mathcal{F}_{\alpha \rightarrow \theta}^{-1}\left\{\frac{\mathcal{F}_{\theta \rightarrow \alpha}\{\tilde{y}(\theta)\}}{\left|\mathcal{F}_{\theta \rightarrow \alpha}\{\tilde{y}(\theta)\}\right|}\right\}
$$

The corresponding flowchart is exposed in Fig. 4(b).

\subsubsection{The generalized synchronous average (GSA)}

The GSA has been proposed as an extension of the synchronous average traditionally applied in stationary regime to the case of nonstationary regime [26]. Recognizing that the signal statistics are dependent on the operating speed the idea is to perform the averaging operation on cycles that belong to the same speed. Formally speaking, the GSA of a signal $y(\theta)$ with angular period $\Theta$ and operating under the speed profile $\omega(\theta)$ is defined as

$$
m_{y}(\bar{\theta}, \omega)=\lim _{\delta \omega \rightarrow 0 \operatorname{card}\left\{K_{\bar{\theta}, \omega}\right\} \rightarrow \infty} \frac{1}{\operatorname{card}\left\{K_{\bar{\theta}, \omega}\right\}} \sum_{k \in K_{\bar{\theta}, \omega}} y(\bar{\theta}+k \Theta)
$$

where $\delta \omega$ stands for the speed resolution and $\mathrm{K}_{\bar{\theta}, \omega}=\{k \in \mathbb{N} \omega-\delta \omega / 2 \leq \tilde{\omega}(\bar{\theta}+k \Theta)<\omega+\delta \omega / 2\}$ stands for the set of samples located at $\bar{\theta}$ and coinciding with speed values in the interval $[\omega-\delta \omega / 2 ; \omega+\delta \omega / 2]$. Note that the GSA is in general a bivariable function of the angle and speed. The (synchronous) deterministic component associated with the speed profile $\omega(\theta)$ is returned by

$$
m_{y}(\theta)=m_{y}(\bar{\theta}=\lfloor\theta \mid \Theta\rfloor, \omega=\tilde{\omega}(\theta)),
$$


a)

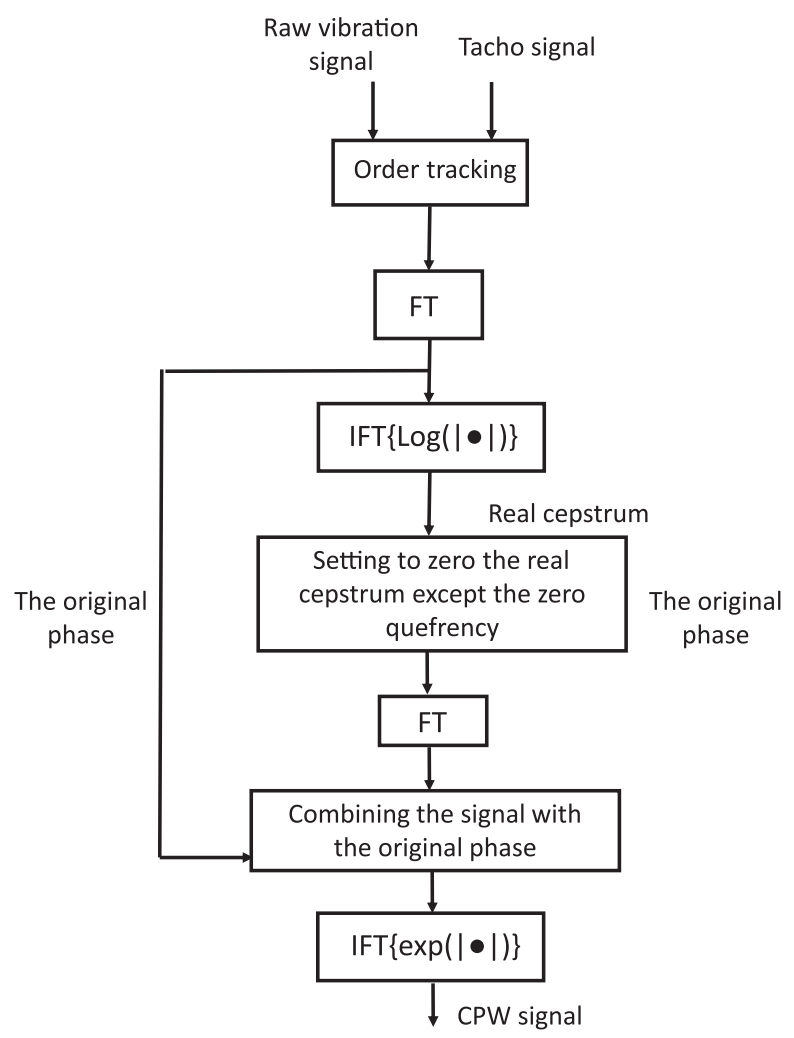

b)

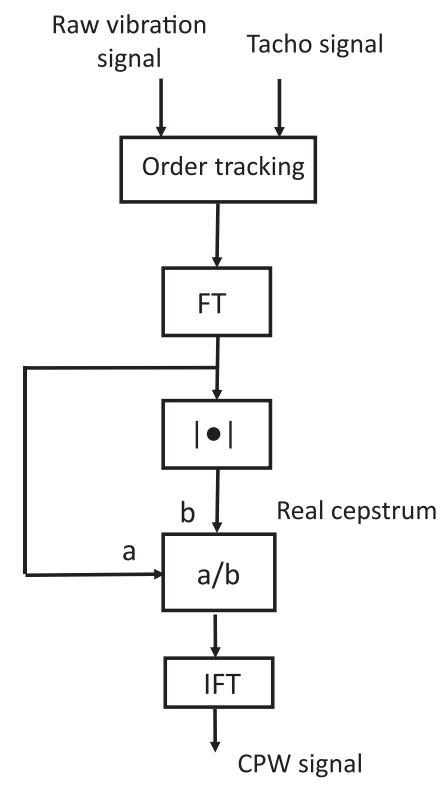

Fig. 4. Flowcharts of the CPW: (a) based on the CPW definition and (b) an equivalent and simpler code.

which is a function of $\theta$ only. The application of the GSA on the vibration model of Section 2.2.1 returns,

$$
m_{x}(\bar{\theta}, \omega)=\sum_{\lambda_{i} \in \Lambda} d^{\lambda_{i}}(\omega) e^{j_{\lambda_{i}} \bar{\theta}},
$$

provided that $\Theta=2 \pi / \lambda_{1}$ and $\lambda_{i}=i . \lambda_{1}$ for all $i=2,3 \ldots$ (see the proof in Appendix A). Interestingly, Eq. (28) perfectly returns the deterministic part:

$$
m_{X}(\theta)=\sum_{\lambda_{i} \in \Lambda} d^{\lambda_{i}}(\tilde{\omega}(\theta)) e^{j^{\lambda_{i} \theta}=\mathrm{d}(\mathrm{t}(\theta))} .
$$

The practical implementation of the GSA can be made via the raw estimator (see Appendix B and Fig. 5(c)).

\subsubsection{CPW or GSA?}

Despite its simplicity and its remarkable ability to blindly eliminate the deterministic component, the CPW technique returns a whitened signal which does not constitute, in itself, the real residue. On the one hand, the whitened signal loses its absolute magnitude, which may be detrimental to diagnostics. On the other hand, the whitening operation distorts the random part in which the CNS component related to the SOI (e.g. a REB fault) is embedded. Moreover, the whitening operation is made in the order domain wherein the dynamic information is effectively lost. This may result in uncontrolled amplification of low signal-to-ratio (SNR) bands, thus increasing the background noise in the SES. Interestingly, the GSA is able to systematically extract the deterministic component without affecting the CNS component. But, this technique has also some disadvantages. First, it needs (relatively) long signals as (i) the speed resolution $\delta \omega$ should be chosen small enough to decrease the estimator bias and (ii) the number of averages should be sufficiently high to decrease the estimator variance. Note that the variance is inversely proportional to the power of the random part [26]. Second, it is more complex than the $\mathrm{CPW}$ as it requires more operations as well as the choice of adequate parameter settings. Third, separate operations of different angular sampling may be needed for multiple families of harmonics. Fourth and last, each of these families should have a commensurate and known angular period. In other words, the GSA is unable to remove modulation sidebands if their angular period is incommensurate with the carrier period. Such configurations, for instance, are frequently met in planetary gearboxes [36]. To conclude, the preference between the CPW and the GSA is generally dependent on the nature of the application, recognizing that the former is likely to cover much more configurations than the latter. 


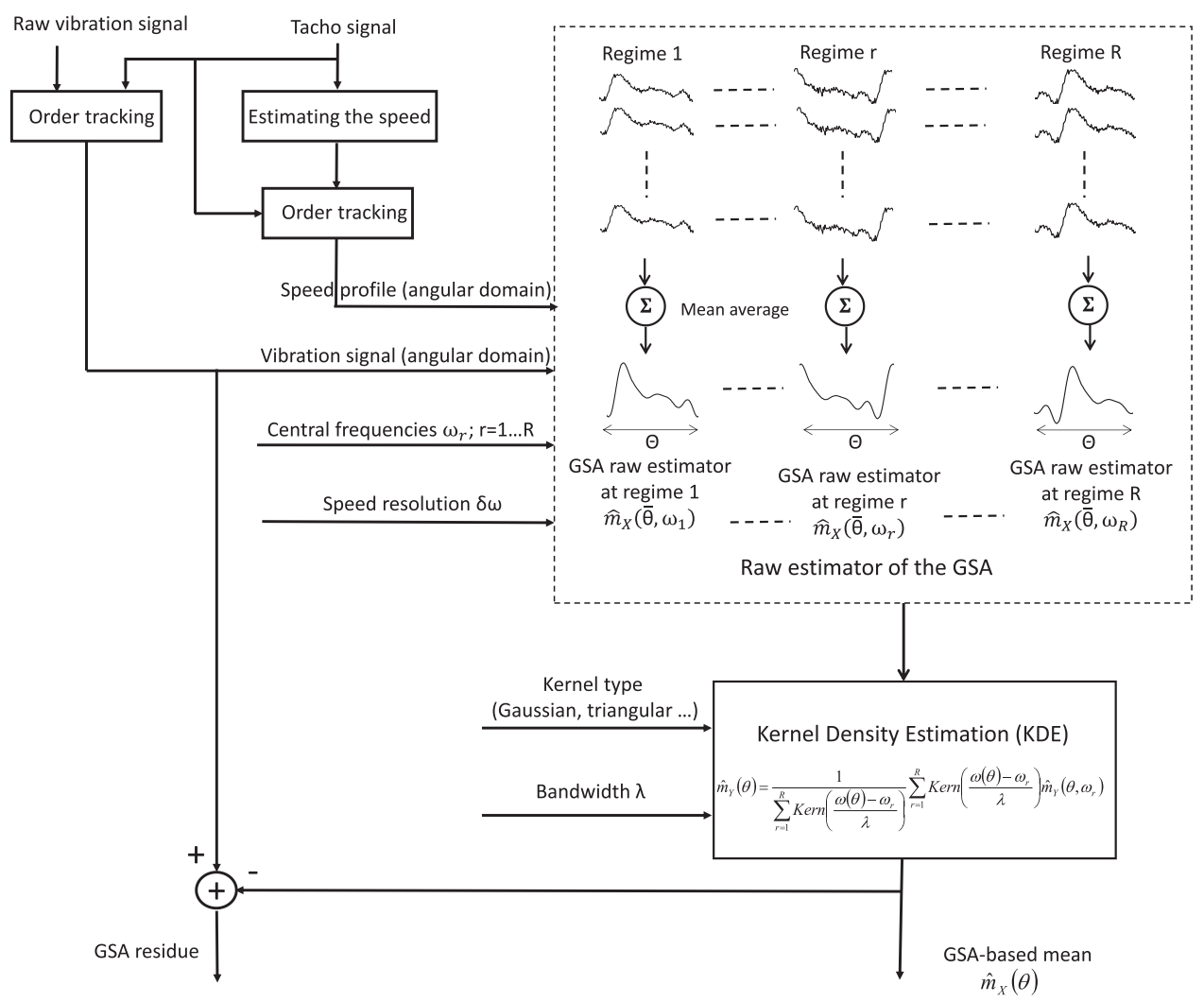

Fig.5. Flowchart of the GSA.

\subsection{Enhancing the source of interest}

In this paragraph, a general envelope enhancement methodology which combines a time-domain filtering operation with either the GSA or the CPW techniques is revised and adjusted.

\subsubsection{Time-filtering operation}

According to Eq. (9), each CNS component is characterized by (i) its cyclic order set which reflects the kinematic of the excitation mechanism associated with the corresponding source and (ii) the spectral content of its carrier which jointly depends on the spectral content of the excitation and on the dynamical properties of the transfer function from the source to the accelerometer. Since the final goal is to detect the signature of the SOI in the envelope, the former can be enhanced by means of a time band-pass filter $f(t)$. Back to the signal/noise decomposition in Eq. (3), the filtered signal reads

$$
\tilde{x}(t)=(s(t)+\mathrm{n}(t)) \otimes f(t)=\tilde{s}(t)+\tilde{n}(t),
$$

where $\otimes$ denotes convolution, $f(t)$ denotes the band-pass filter, $\tilde{s}(t)$ and $\tilde{n}(t)$ provide respectively an image of the signal of interest and the noise. Ideally, the filter should be conceived to maximize the SNR, i.e.

$$
\underset{f(t)}{\operatorname{argmax}}\left\{\lim _{T \rightarrow \infty} \frac{\frac{1}{T} \int_{-T / 2}^{+T / 2}|\tilde{s}(t)|^{2} d t}{\frac{1}{T} \int_{-T / 2}^{+T / 2}|\tilde{n}(t)|^{2} d t}\right\} .
$$

Optimally, the energy of $\tilde{n}(t)$ would become negligible as compared to that of $\tilde{s}(t)$. In this case, the resulting SES

$$
\operatorname{SES}_{\tilde{x}}(\alpha) \approx \operatorname{SES}_{\tilde{s}}(\alpha)=\sum_{\gamma_{i}^{(s)} \in \Gamma_{S}} \operatorname{SES}_{\tilde{s}}^{\gamma_{\tilde{S}}^{(s)}}\left(\alpha-\gamma_{i}^{(s)}\right),
$$

has a symptomatic discrete distribution strictly at the cyclic orders associated with the SOI (see the proof in Appendix A). 


\subsubsection{Choice of the filter parameters}

As indicated in the last subsection, the filtering operation should be performed around a high-SNR frequency band. In practice, it often happens that the SNR is high around resonance bands. Therefore, a traditional solution is to perform a modal analysis ${ }^{1}$ step to explore the dynamical properties of the system [38]. In general, modal analysis is either based on structural testing (e.g. "hammer impact test" [59]) or finite element analysis-based simulation [60]. The former technique (structural testing) requires a disassembly of the machine to reach the potential excitation point, whereas the latter one (finite element analysis simulation) requires a complex modeling of the system. These practical difficulties have limited the application of such techniques.

There are other blind approaches based on statistical signal processing which consist of finding the band that maximizes a criterion, e.g. impulsivity (e.g. kurtogram) [31,61,62,63] or cyclostationarity (e.g. spectral coherence) [17]. However, these approaches rely on the assumption of constant operating speed, thus their applicability is jeopardized in VSC. The recent advances in the CS theory [29] have made now possible to exploit more sophisticated techniques that can adapt with the operating speed variability. In particular, the angle/time cyclostationary (AT-CS) theory has allowed the extension of the spectral coherence, to the so-called "order frequency spectral coherence" [16]. The squared magnitude of the latter, when evaluated at the cyclic orders of a CNS signal, can be seen as a measure (normalized between 0 and 1 ) of the signal-to-noise ratio along the spectral frequency band.

\subsubsection{An envelope enhancement methodology}

A trivial methodology to enhance a cyclo-non-stationary SOI in a raw signal is to remove first the deterministic component and then filter the obtained signal in the time domain around a high-SNR band. This methodology consists of the following steps:

1) Remove the deterministic component using the CPW or GSA in the angular domain.

2) Go back to the time domain.

3) Band-pass filter the signal around a high-SNR frequency band.

4) Order track the signal (go again to the angular domain).

5) Compute the SES.

Note that a similar procedure was proposed in [28], yet the synchronous average was used instead of the GSA.

\section{Numerical evaluation}

This section validates the theoretical analysis, the different envelope enhancement approaches and the qualitative comparisons on simulated gear and bearing vibration signals. First, the corresponding models are given and their contributions in the SES are investigated. Second, a comparison between the ISA, the CPW and the GSA is provided. Third, the envelope enhancement methodology provided in 3.2.3 is applied to enhance and separate the CNS sources.

\subsection{Vibration signal model in variable speed conditions}

The aim of this subsection is to simulate a vibration signal of a typical powertrain constituted by a parallel gearbox and a faulty REB. The input wheel comprises $Z_{1}=56$ teeth and the output wheel $Z_{2}=60$ teeth. The former has a local defect in one of its teeth, whereas the latter is subjected to a distributed wear fault. The REB is located at the input shaft - the reference shaft - and contains an outer-race local fault with a characteristic ball-pass-order on the outer-race (BPOO) at 2.1 orders - i.e. 2.1 times the reference rotation speed. The deterministic component in the signal is principally induced by the meshing force and the local fault in the input wheel. It appears in the order spectrum as (widened) peaks at the orders $m \cdot Z_{1}+n$ with $m \in \mathbb{Z}, n \in \mathbb{Z}^{*}$. The CNS component is induced by the output gear and the input REB. The energy of the output gear contribution is principally concentrated in a resonance band centered at $4 \mathrm{kHz}$ with bandwidth of $300 \mathrm{~Hz}$; it is expected to be discretely distributed in the SES at the output shaft order - which equals $56 / 60=0.933$ - and its harmonics. The energy of the input gear contribution is principally concentrated in a resonance band centered at $2 \mathrm{kHz}$ with bandwidth of $400 \mathrm{~Hz}$; it is expected to be discretely distributed in the SES at the BPOO and its harmonics. A white noise modulated by a speed-dependent increasing function is also added to the signal to model the background noise. The principal characteristics of the simulated signal are gathered in Table 1, while detailed equations of the model are provided in Appendix C.

The time domain representations of the signals components are displayed in Fig. 6.

The reference speed varies according to a raised-cosine function between $5 \mathrm{~Hz}$ and $15 \mathrm{~Hz}$ over a record duration of $20 \mathrm{~s}-$ equivalent to 198 cycles executed by the reference (which is the input shaft associated with order 1 ); the corresponding profile is displayed in Fig. 7(a). The spectrograms of the different contributions are displayed in Fig. 7(b)-(f). For the REB signal (Fig. 7(b)), an energy increase is perceived at $2 \mathrm{kHz}$ which corresponds to the passage on the resonance frequency. For

\footnotetext{
${ }^{1}$ to estimate the transfer functions from the SOI to the acceleration.
} 
Table 1

Characteristics of the simulated signal $\left(\boldsymbol{f}_{\text {res }}\right.$ : resonance frequency; $\boldsymbol{f}_{\boldsymbol{b}}$ : Bandwidth).

\begin{tabular}{llll}
\hline & Deterministic gear component & Random gear component & REB (local fault) \\
\hline Resonances & $\left\{\begin{array}{l}f_{\text {res }}=3 \mathrm{kHz} \\
f_{b}=2 \mathrm{kHz}\end{array}\right.$ & $\left\{\begin{array}{l}f_{\text {res }}=4 \mathrm{kHz} \\
f_{b}=300 \mathrm{~Hz}\end{array}\right.$ & $\begin{array}{l}f_{\text {res }}=2 \mathrm{kHz} \\
f_{b}=400 \mathrm{~Hz}\end{array}$ \\
Fundamental order & 1 & $56 / 60=0.933$ & 2.1 \\
Energy (\%) & $40 \%$ & $20 \%$ & $\mathrm{X}$ \\
\hline
\end{tabular}
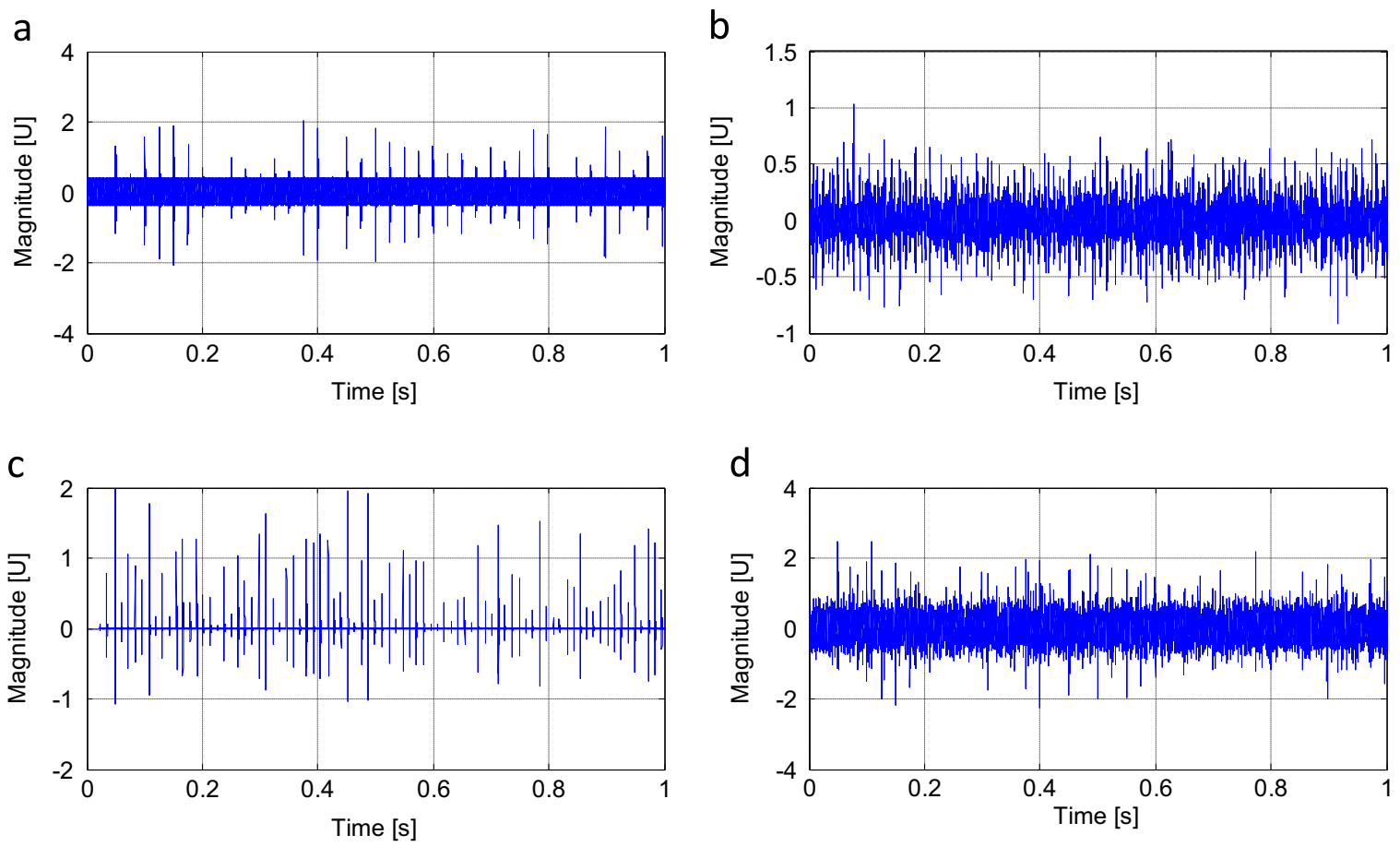

Fig. 6. The time domain representation over $1 \mathrm{~s}$ duration of the simulated signals corresponding to (a) the deterministic gear signal, (b) the random gear signal, (c) the REB signal, (d) the complete vibration signal.

the deterministic gear signal (Fig. 7(c)), the meshing harmonics appear as multiple curves homothetic with the speed profile, while the amplitude of the modulation induced by the local fault remains relatively small. The random gear signal (Fig. 7(d)) shows a continuous increase around the resonance frequency located at $4 \mathrm{kHz}$. The energy of the background noise (Fig. 7(f)) is uniformly distributed along the frequency axis. Last, the spectrogram of the resulting vibration signal (Fig. 7(f)) is dominated by the deterministic gear component as it constitutes $40 \%$ of the signal energy. Note that the energy distribution is intensified in the middle of the record since the speed-dependent amplitude modulation is maximal at this point.

Eventually, the SES is applied on different components of the signal and the obtained results are reported in Fig. 8. The SES of the REB component shows some peaks at the BPOO and its harmonics (see Fig. 8(a)). The SES of the deterministic gear component shows peaks at the reference order and its harmonics (see Fig. 8(b)), while the random gear component shows peaks at the output wheel order (0.933) and its harmonics (see Fig. 8(c)). The SES of the noise does not show any non-zero peak (see Fig. 8(d)). The SES of the random part - constituted of the REB component, the random gear component and the background noise - is obviously equivalent to the superposition of all individual SES since the signal components are assumed uncorrelated (see Fig. 8(e)). In this case, the bearing fault component interferes with the random gear contribution. The problem is aggravated in the SES of the vibration signal as the deterministic component also contributes to the SES (see Fig. 8(f)). These preliminary results confirm the theoretical analysis of Section 2.

\subsection{Removing the deterministic part}

The goal of this subsection is to investigate the performance of the ISA, the GSA and the CPW in removing the deterministic component. In the following, the ISA is applied on the signals with a reference period equal to $2 \pi$ (i.e. equivalent 
a

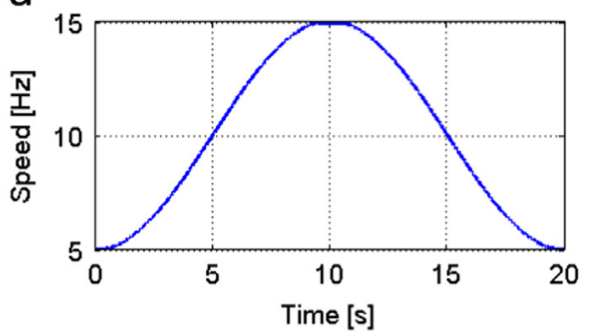

C

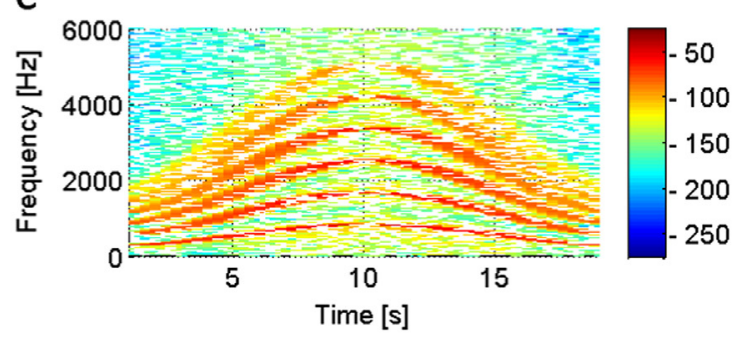

e

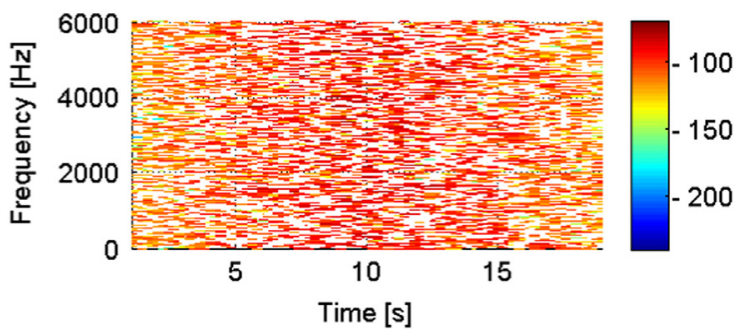

b
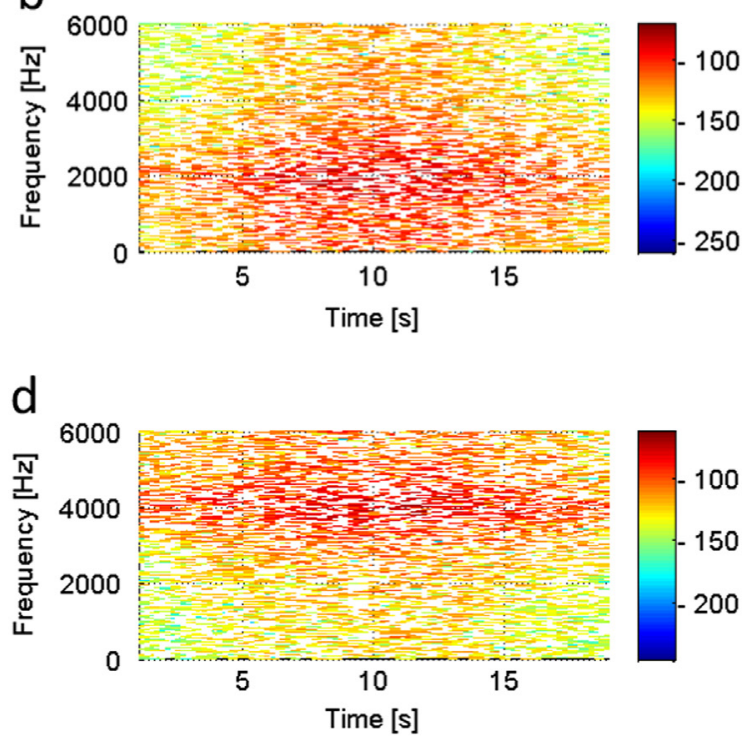

f

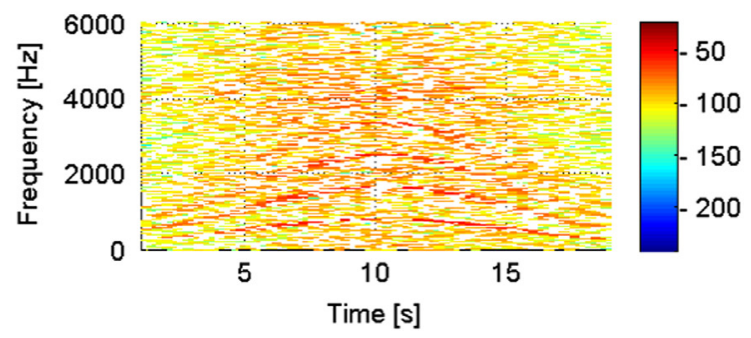

Fig. 7. (a) Speed profile of the reference. Spectrograms of (b) the REB signal, (c) the deterministic gear signal, (d) the random gear signal, (e) the background noise and (f) the complete vibration signal.

to the input gear order). The first meshing harmonic - located at the order 56 - is used for demodulation and the filter bandwidth is visually estimated according to the existing energy leakage (the filter bandwidth is taken equal to 0.2 order). The demodulated harmonic and the equivalent order fluctuations with which the phase corrections are performed are displayed in Fig. 9(a) and (b), respectively. Next, the GSA is performed on acceleration signals to eliminate the gear component. Evidently, the reference period used in the average is also chosen equal to the input gear rotation. The speed profile is discretized with concatenated intervals of speed resolution equal to $0.4 \mathrm{~Hz}$ with $50 \%$ overlap. The order spectra of the resampled signal, the ISA residue and the GSA residue are displayed in Fig. 9(c) and (d). The ISA suppresses a part of the deterministic gear component energy, whereas the GSA evidences almost total suppression. Afterwards, the CPW is applied on the original signal. The SES of the original signal, the ISA residue, the GSA residue and the CPW signal are computed for both profiles and reported in Fig. 10. As expected, the SES of the raw signal evidences several misleading harmonics of deterministic origin at the integer orders (see Fig. 10(a)), whereas slight enhancement is perceived after removing the ISA (see Fig. 10(b)): the amplitudes of these harmonics are attenuated but still exceeds those of the CNS components. Noticeably, the SES of the GSA residue (see Fig. 10(c)) and the CPW signal (see Fig. 10(d)) witness a complete absence of these components, yet the absolute value of the latter is totally lost. Moreover, by comparing the obtained SE spectra (see Fig. 10(c) and (d)) to the SES of the random part (see Fig. 8(e)), a significant distortion in the random part is seen in the CPW case caused by the whitening operation. In particular, the first REB fault harmonic (at the order 2.1) was the largest in the SES of the random part, while that of the output gear wear (at the order 0.933 ) becomes the largest in the CPW case.

\subsection{Separating CNS components}

Despite their ability to reduce deterministic interferences, the GSA and the CPW were not able to clearly reveal the fault signature of neither the REB nor the output gear. Assuming a prior knowledge of the excited resonances, the methodology proposed in Section 3.2.3 is applied to the signal to separate these two contributions. For the REB case, the central frequency of the filter is chosen equal to the corresponding resonance frequency (i.e. $2 \mathrm{kHz}$ ), while the filter bandwidth is set to $600 \mathrm{~Hz}$. The obtained SE spectra are displayed in Fig. 11 (a) and (b), respectively referring to GSA-based and CPW based 
a
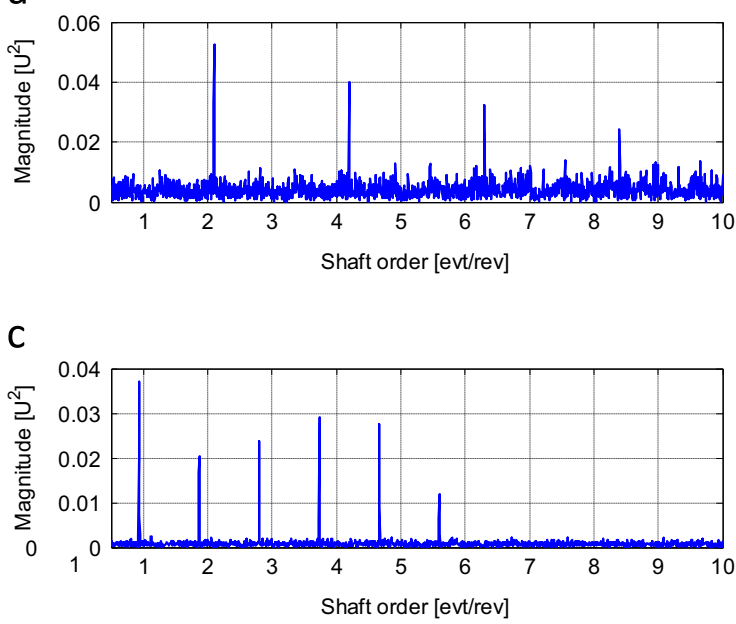

e

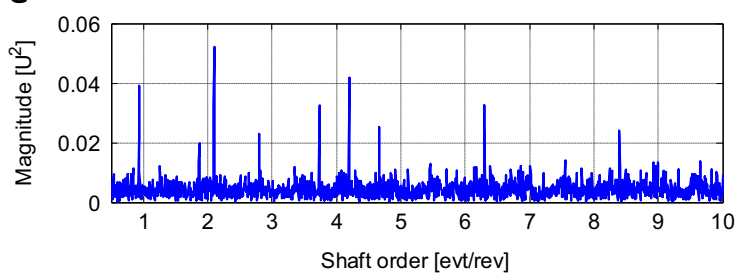

b

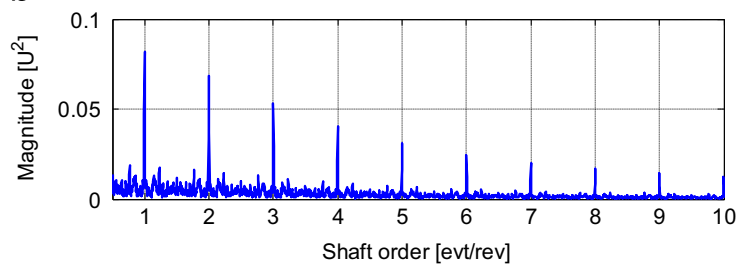

d

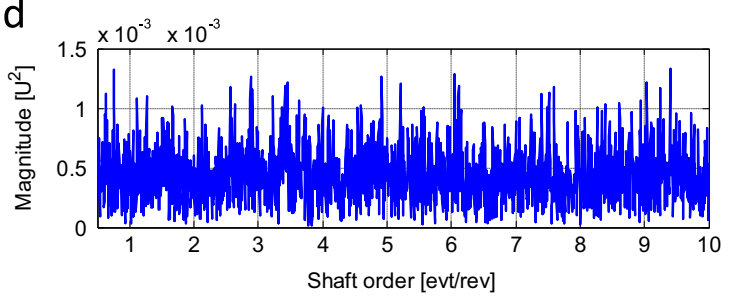

$f$

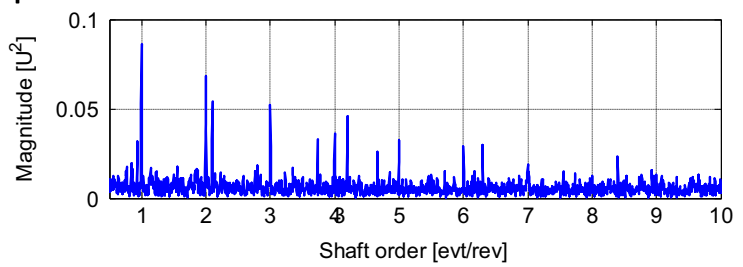

Fig. 8. SES of (a) the REB signal, (b) the deterministic gear signal, (c) the random gear signal, (d) the background noise, (e) the random part and (f) the complete vibration signal.

a

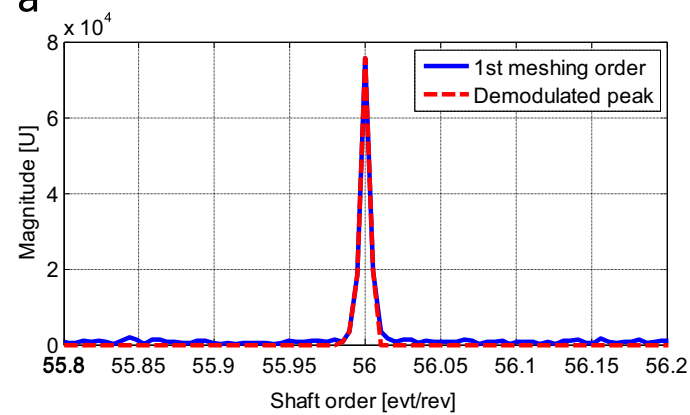

C

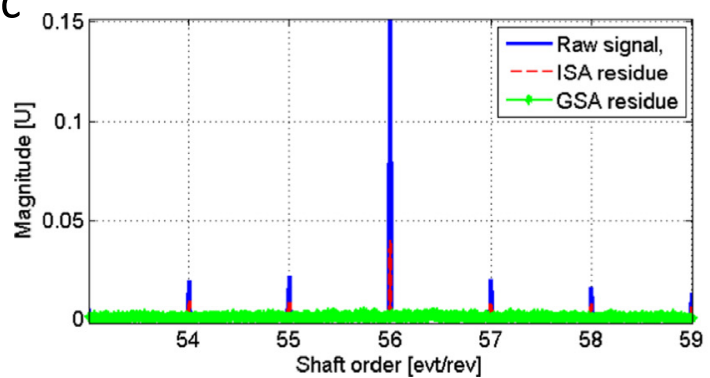

b

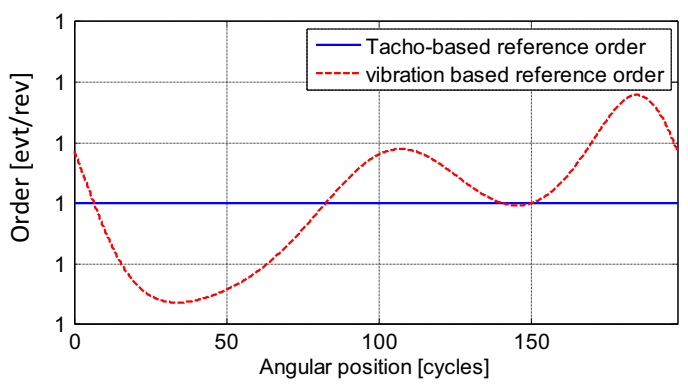

d

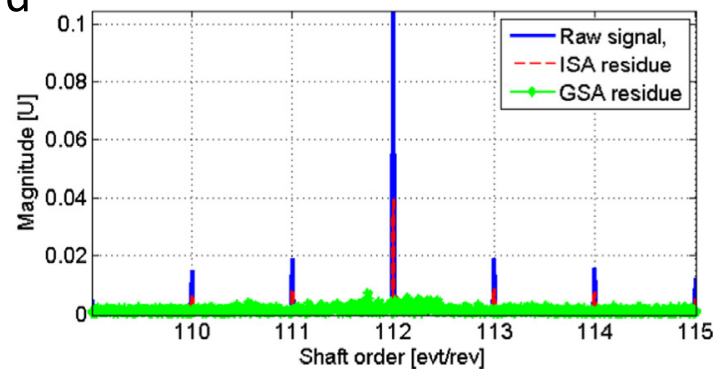

Fig. 9. (a) The harmonic used for demodulation and (b) the equivalent order fluctuations used for phase correction. Comparison of the (amplitude) order spectra of the resampled signal, the ISA residue and the GSA residue: close-ups around (c) the fundamental and (d) the second meshing harmonics.

strategies. Interestingly, the REB contribution is successfully enhanced and its signature becomes much clearer in the SES: the remaining peaks are strictly located at the BPOO and its harmonics. A bandpass filter was also applied to enhance the output gear contribution, with central frequency chosen equal to the corresponding resonance frequency (i.e. $4 \mathrm{kHz}$ ) and 
a

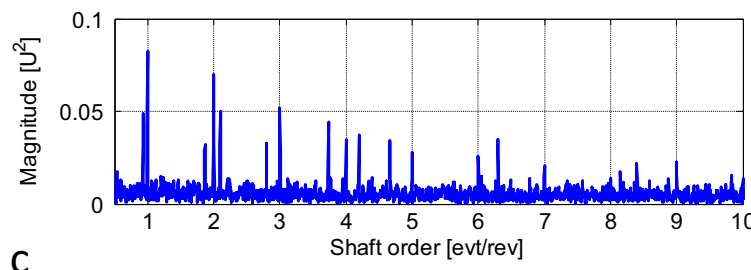

C

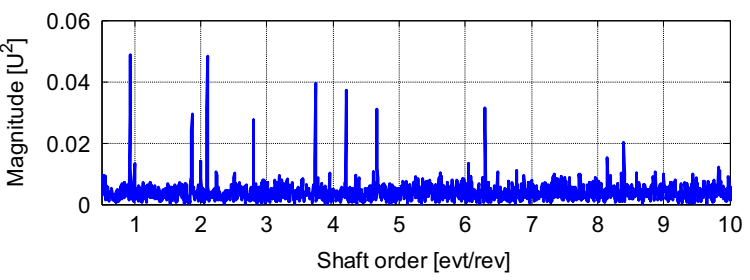

b
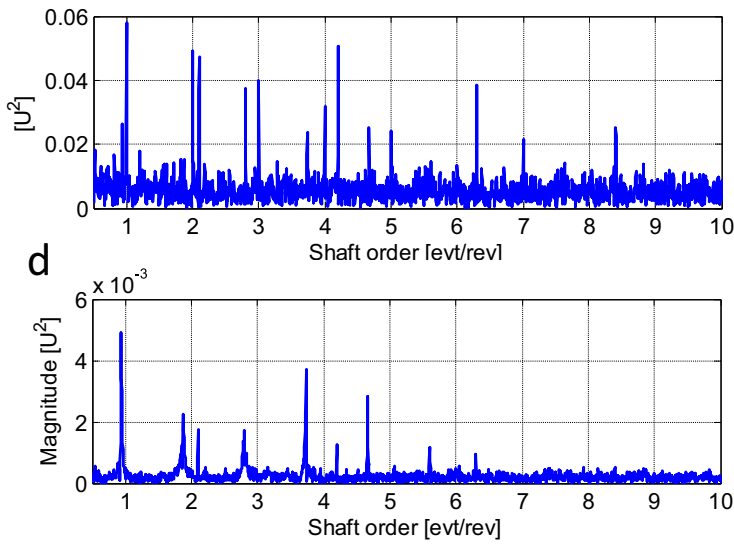

Fig. 10. SES of (a) the original signal, (b) the ISA residue, (c) the GSA residue and (d) the CPW signal. The targeted SES is displayed in Fig. 8(e).

a

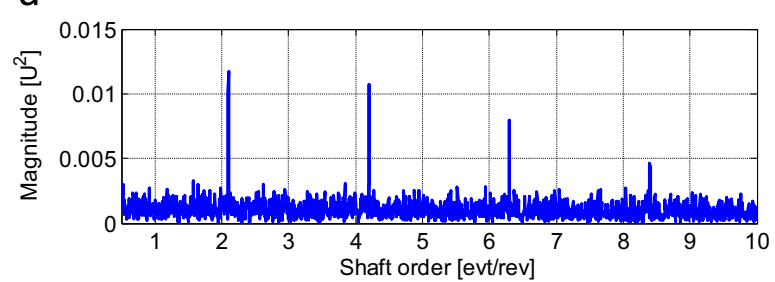

C

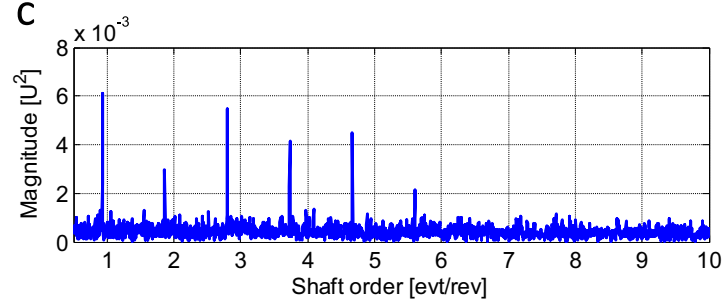

b
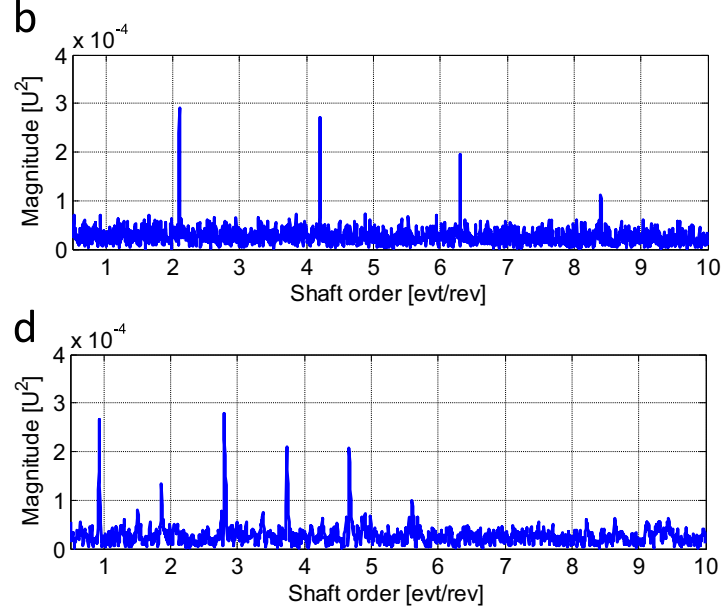

Fig. 11. Envelope enhancement procedure applied on the simulated signal with filter parameters $\mathbf{f}_{\mathbf{c}}=2 \mathbf{k H z}$ and $\mathbf{B w}=600 \mathbf{H z}$ : (a) GSA-based strategy and (b) CPW-based strategy; filter parameters $\mathbf{f}_{\mathbf{c}}=4 \mathbf{k H z}$ andBw $=600 \mathbf{H z}$ : (c) GSA-based strategy and (d) CPW-based strategy. The targeted SES is displayed in Fig. 8(c).

bandwidth set to $600 \mathrm{~Hz}$. The obtained SE spectra are displayed in Fig. 11(c) and (d), respectively referring to a GSA-based and CPW-based strategies. The output gear contribution is successfully enhanced and its signature becomes much clearer in the SES: the remaining peaks are strictly located at the output gear order and its harmonics. It is worth noting that the filtering operation decreases the distortion of the cyclic components. This is due to the fact that the SNR is almost constant over the filtered band, so that the distortion affects the energy of the entire cyclic content. As a consequence, all the corresponding peaks in the SES will be attenuated uniformly. Evidently, the distortion in the signature is expected to be lower for narrow band filters.

\section{Experimental evaluation}

The principal object of this section is to experimentally validate the theoretical analysis and qualitative comparisons on real-world gear and REB vibration signals. It reports a typical situation in the field of rotating machine diagnostics, where deterministic and random gear related components contribute to the SES and mask the REB fault signature - originally random. 


\subsection{Test rig and experiments}

The test rig under study is located at Cetim $^{2}$ and depicted in Fig. 12. It essentially comprises an asynchronous motor supplied by a variable-speed drive to control the motor speed, followed by a spur gear with 18 teeth and ratio of 1 . Gears are subjected to excessive wear. This returns an expected meshing signature at 18 order - i.e. 18 times the rotation speed - with potential sidebands spaced by 1 order. Two identical REBs are installed behind the spur gear; the healthy one is closer to the gears than the faulty one which is coupled to an alternator by means of a belt to impose a constant load. The characteristics of the REBs are as follows: ball diameter $=6 \mathrm{~mm}$, pitch diameter $=25 \mathrm{~mm}$, number of elements $=8$. This returns an expected ball-pass-order on the outer-race (BPOO) at 3.04 orders. An optical keyphasor of type "Brawn" is fixed close to the motor output to measure the rotational shaft position. In addition, two accelerometers, hereafter denoted as "Acc1" and "Acc2", are located on the healthy and faulty REBs in the vertical direction to measure the produced vibrations. The sampling rate is set to $25.6 \mathrm{kHz}$. Two experimental tests are conducted. In the first one, a runup from 903 to $1428 \mathrm{rpm}$ (see Fig. 13(a)) is imposed to the electric motor during $15 \mathrm{~s}$ - equivalent to 285 cycles executed by the machine. The acquired signals are illustrated in Fig. 13 (b) and (c) referring to Acc1 and Acc2, respectively. In the second experiment, a random speed profile ranging between 704 and 2514rpm (see Fig. 13(d)) is imposed to the electric motor during 15s- equivalent to 392 cycles executed by the machine. The acquired signals are illustrated in Fig. 13 (e) and (f) referring to Acc1 and Acc2, respectively.

The AT-CS analysis of this bench has been performed in a previous contribution [16] in the case of stationary and nonstationary operating condition. Valuable information regarding the high-SNR spectral band of the CNS components emitted by the faulty REB and the gear wear were returned by the order-frequency spectral coherence. These information are summarized in Table 2 and will be subsequently used to test the envelope enhancement methodology in Sections 5.2 .2 and 5.3.2.

\subsection{Case study 1: the REB component dominates the random part}

This paragraph treats the case where the accelerometer is mounted on the faulty REB (Acc2) and its component dominates the random part. At first, this case is exploited to compare the ISA, the CPW and the GSA for the two profiles according to their capability to improve the SES. Last, the REB component will be enhanced by the envelope enhancement methodology to return a clear presentation of its signature in the SES.

\subsubsection{Removing the deterministic gear contribution}

This subsection compares the effectiveness of the ISA, the CPW and the GSA in removing the deterministic gear component and in enhancing the SES.

First, the ISA is applied on Acc2 signals of both experiments. Since the gears have a unit ratio, the reference period to be used for the average in the ISA is chosen equal to the gear rotation, which makes possible to account for gear modulations. The order spectra were visually inspected to choose the higher meshing harmonic for demodulation and to estimate the filtering bandwidth according to the existing energy leakage. The demodulated harmonics and the equivalent order fluctuations (see in Eq. (18)) with which the phase corrections are performed are displayed in Fig. 14. Contrary to the random speed-varying case, the second meshing order is more energetic than the first one in the runup case and thus was chosen for demodulation. Note that the filter bandwidth is chosen larger in the random speed-varying ( 0.1 order) case than that in the runup case ( 0.02 order). This is explained by the fact that the high variability of the speed in the latter increases the energy leakage at the synchronous order and, thus, a wider filter is needed to filter out the component. This confirms the theoretical analysis performed in Section 2.2.1.

Next, the GSA is performed on acceleration signals to eliminate the gear component. The reference period used for the average in the GSA is also chosen equal to the gear rotation. The speed profile is discretized with concatenated intervals of speed width equal to $30 \mathrm{rpm}$ with $50 \%$ overlap. The order spectra of the resampled signal, the ISA residue and the GSA residue are displayed in Fig. 15. The ISA eliminates a part of the gear contribution energy in the runup case, yet it completely fails in the random speed-varying case as the corresponding residues still contain high energy components around the synchronous orders. Note that, in the latter case, the enhancement was principally perceived at the demodulated peak (i.e. 1st meshing order - see Fig. 15 (d)). This is believed to be due to the fact that, unlike the random speed-varying case, the runup profile varies in a low-speed operating margin that does not include structural resonances. The failure is principally induced by (i) the passage of the gear component in structural resonance bands and (ii) the ignorance of long-term energy modulations. Evidently, these effects are magnified in the random speed-varying case since the speed varying margin is higher and, consequently, the induced modulations are greater. On the contrary, the GSA successfully eliminates the gear component in both cases, ensuring an excellent performance even under large and random speed variations.

Finally, the CPW is applied on the acceleration signals of both profiles. The SES of the resampled (raw) signal, the ISA residue, the GSA residue and the CPW signal are computed for both profiles and displayed in Fig. 16. As expected, the SES of the raw signal evidences several misleading harmonics synchronous with the reference shaft order (see Fig. 16 (a) and (e)), whereas slight enhancements are perceived after removing the ISA (see Fig. 16 (b) and (f)). With the exception of the first

\footnotetext{
${ }^{2}$ Technical Center for Mechanical Industries - Senlis, France.
} 


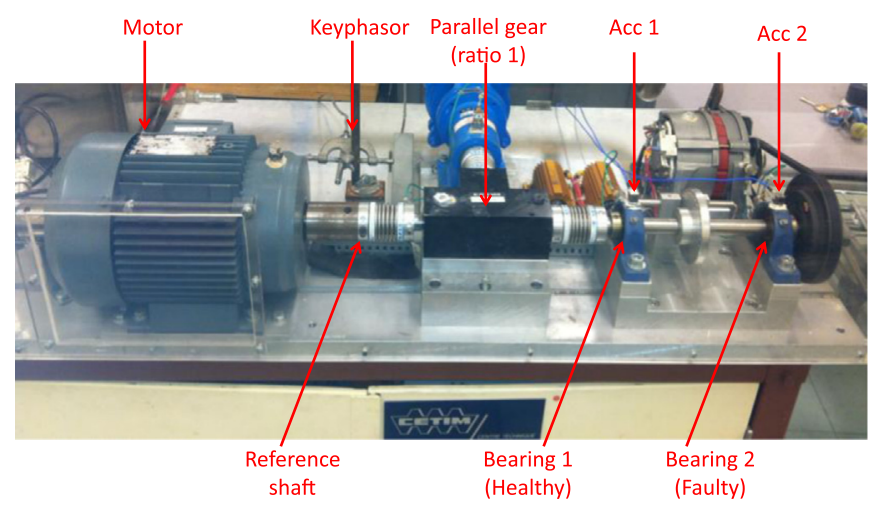

Fig. 12. The test bench located at Cetim (Senlis, France).

a

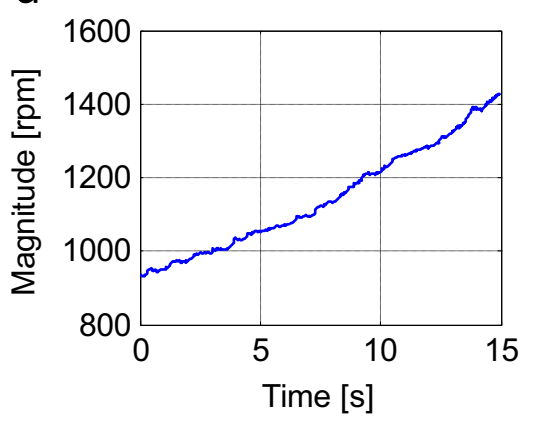

d

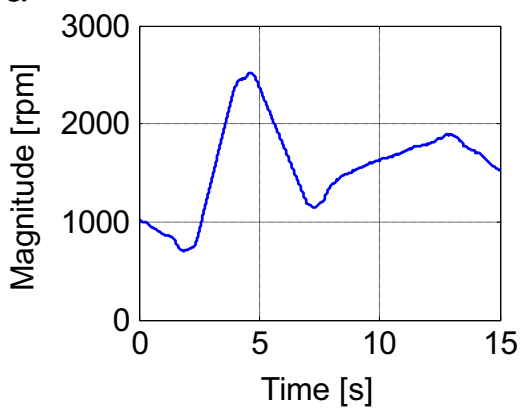

b

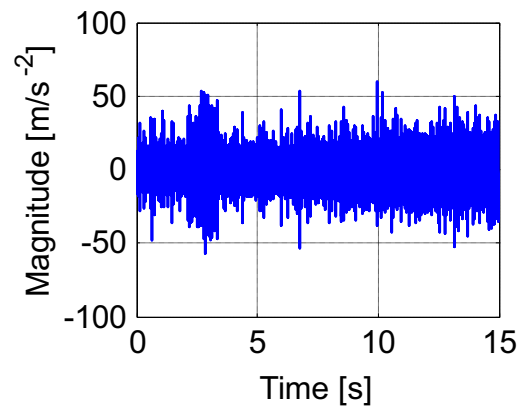

e

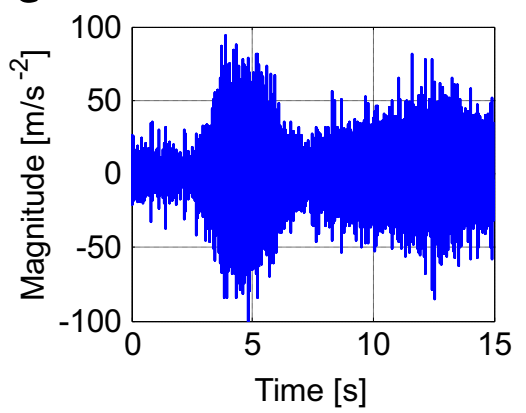

C

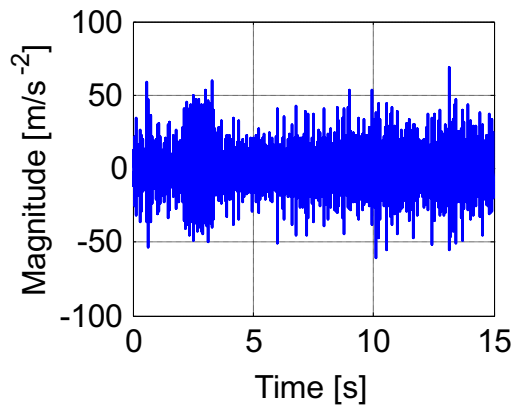

f

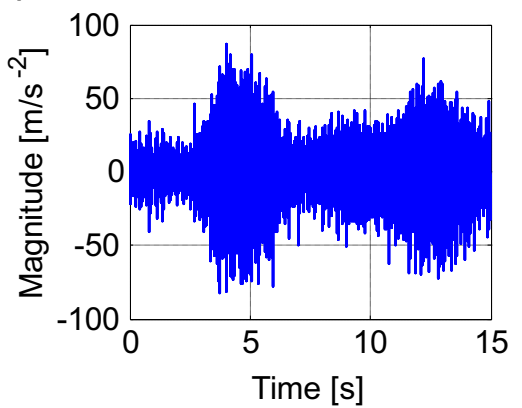

Fig. 13. Experiment 1: (a) runup profile, (b) Acc1 signal and (c) Acc2 signal. Experiment 2: (d) random speed-varying profile, (e) Acc1 signal and (f) Acc2 signal.

Table 2

Principal high-SNR bands of the CNS vibration signals emitted by the faulty REB and the gear, and measured by Acc1 and Acc2. These information are based on the AT-CS analysis performed in Ref. [16].

\begin{tabular}{cll}
\hline & Acc1 & Acc2 \\
\hline High-SNR bands & {$[1.5 \mathrm{kHz} ;$} & $2.5 \mathrm{kHz}]$ \\
(faulty REB) & {$[8 \mathrm{kHz} ; 11 \mathrm{kHz}]$} & \\
High-SNR bands & {$[6 \mathrm{kHz} ; 8 \mathrm{kHz}]$} & $2.5 \mathrm{kHz}]$ and \\
(gear wear) & {$[8 \mathrm{kHz} ; 11 \mathrm{kHz}]$} & \\
\hline
\end{tabular}

two harmonics, the CPW significantly attenuates the gear related peaks (see Fig. 16 (a) and (e)), yet presenting three disadvantages. The first one is related to the increase of the noise level owing to the amplification of low signal-to-ratio spectral bands. The second one is the distortion of the REB contribution. Precisely, an attenuation of the BPOO peak relative to the second gear harmonic (to be compared with that of the GSA) is noticeable in the runup case, while a complete 

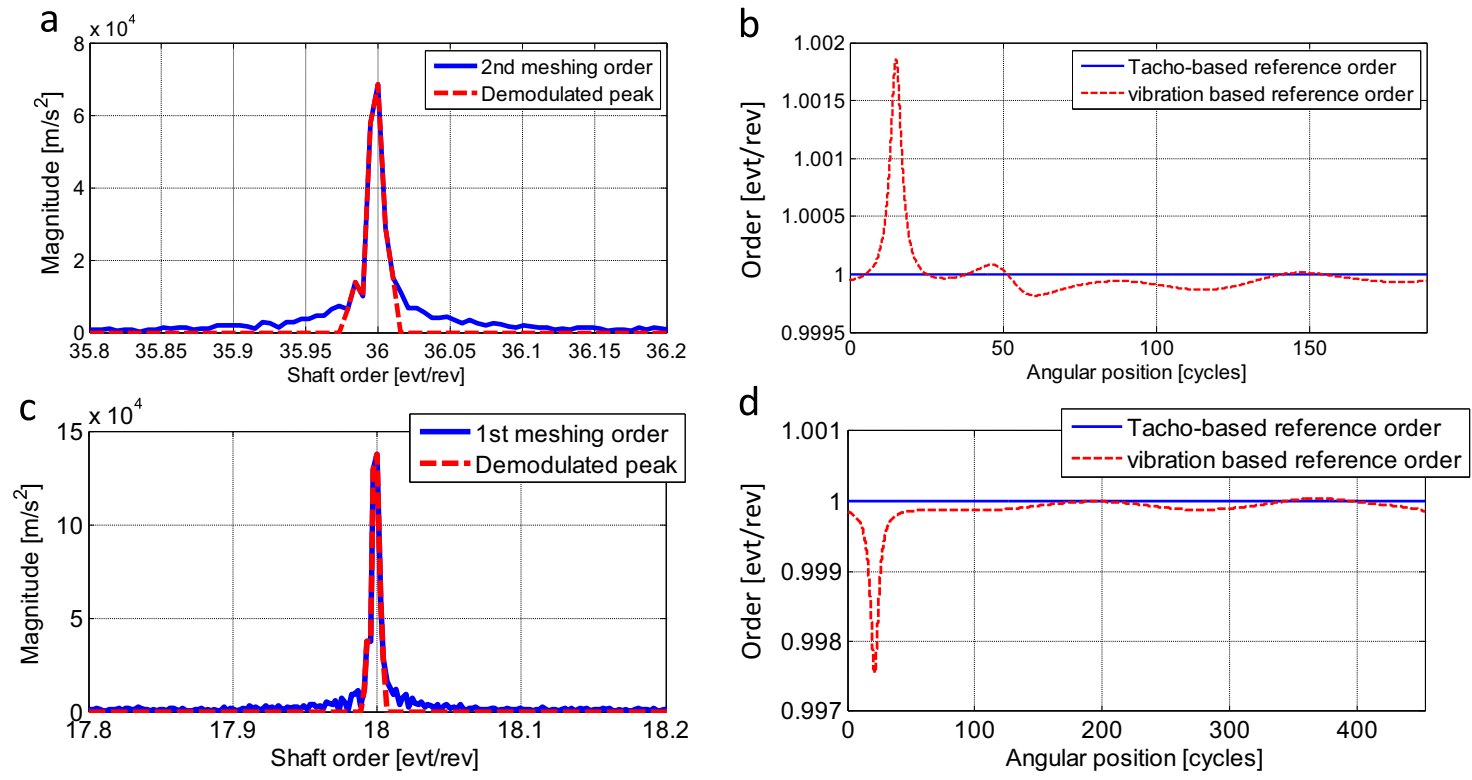

Fig. 14. Runup case (Acc2 signal): (a) the harmonic used for demodulation and (b) the equivalent order fluctuations used for phase correction. Random speed-varying case: (c) the harmonic used for demodulation and (d) the equivalent order fluctuations used for phase correction.

a
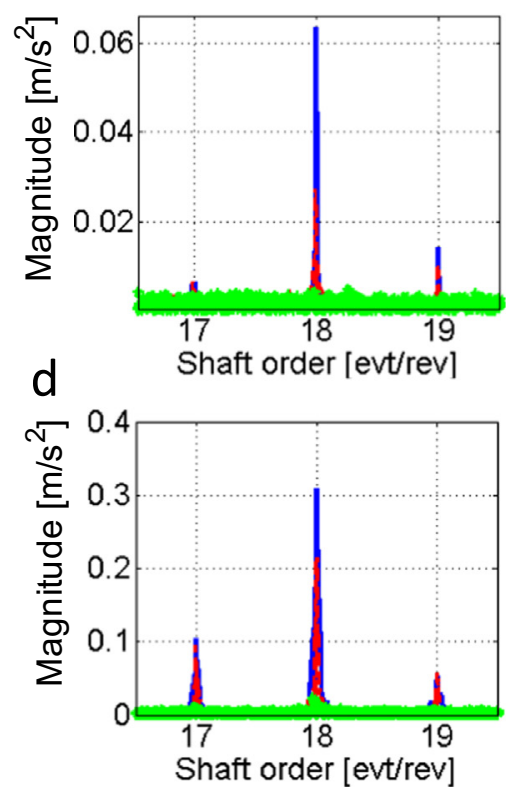

b

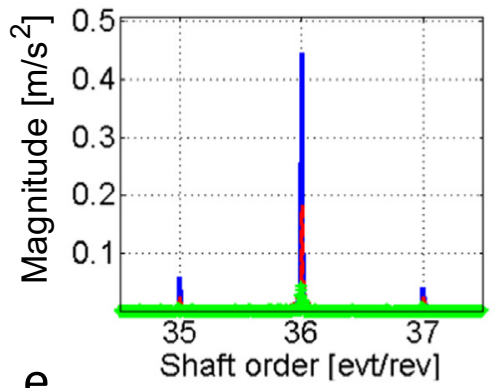

e

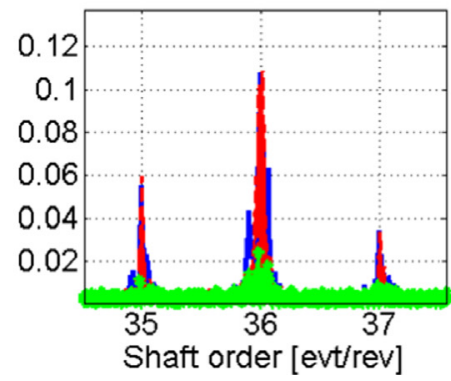

C
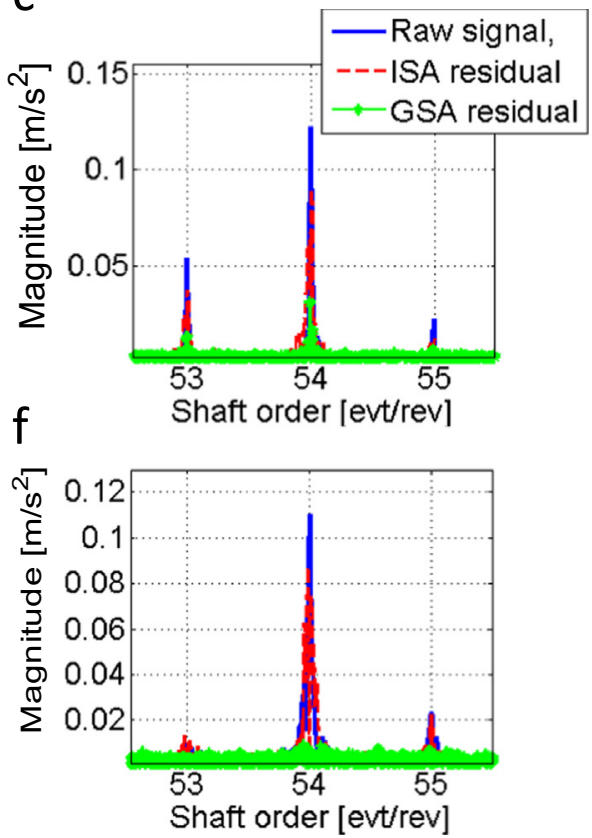

Fig. 15. Comparison of the (amplitude) order spectra of the resampled signal, the ISA residue and the GSA residue (see the legend in the upper right box) on Acc2 signal; (i) in runup1 case: close-ups around (a) the fundamental, (b) the second and (c) the third harmonic of meshing order; (ii) in runup2 case: close-ups around (d) the fundamental, (e) the second and (f) the third harmonic of meshing order.

disappearance of the second REB fault harmonic (note the disappearance of the order 6.08 in Fig. 16 (h)) is observed in the random speed-varying case. Seemingly, the CPW technique loses its efficiency as the speed variability increases. The third disadvantage is the total loss of the absolute value of the fault peaks. Noticeably, the SES of the GSA residue evidences much better the REB signature (see Fig. 16 (c) and (g)), preserving the magnitude of the fault harmonics.

Note that some peaks at the gear rotation order and harmonics remain in the SES. These components are random in nature and generated by gear wear [16], as opposed to the traditional deterministic nature of gear vibrations (e.g. meshing, a crack or spall fault, etc.) [10]. 

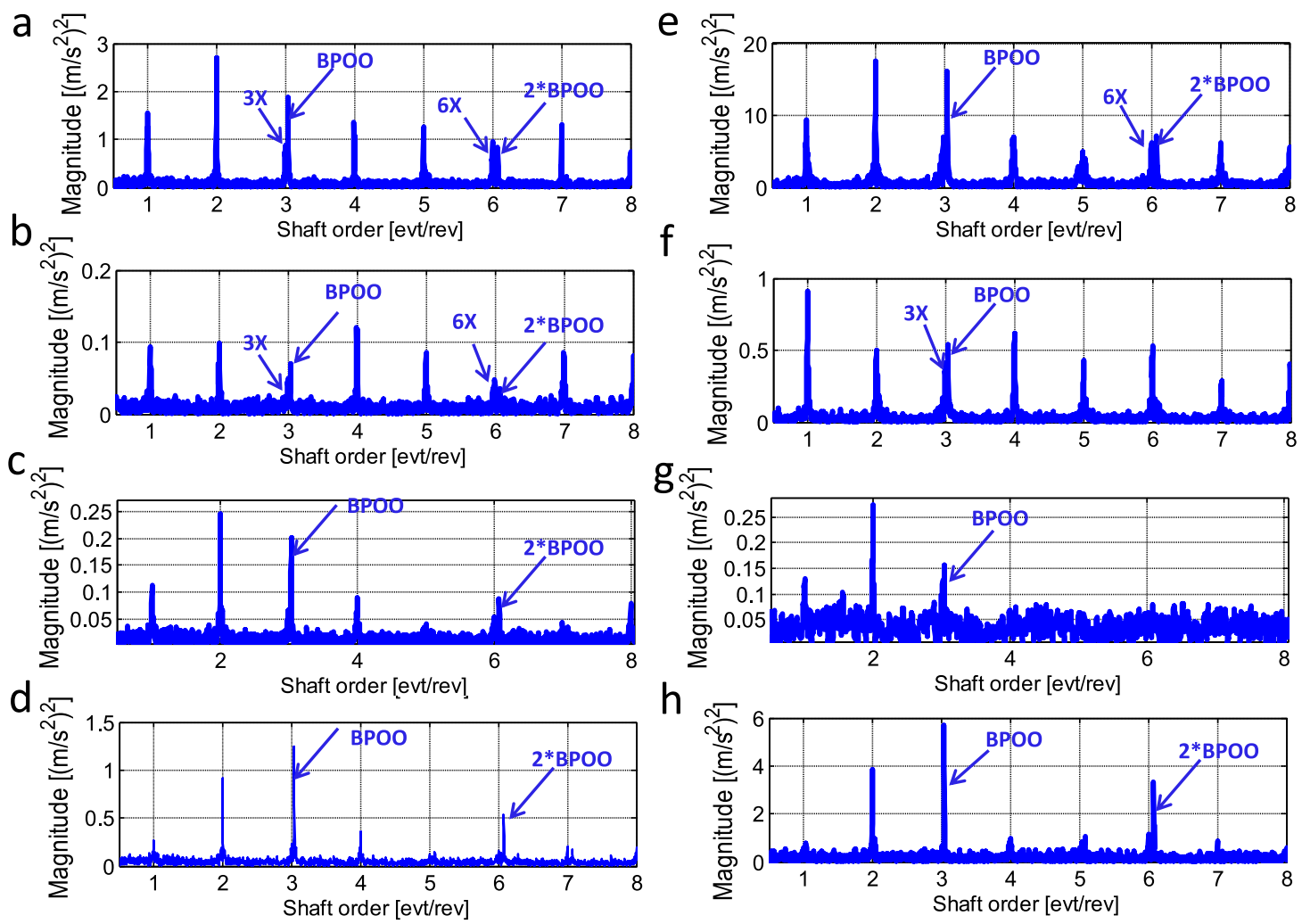

$f$
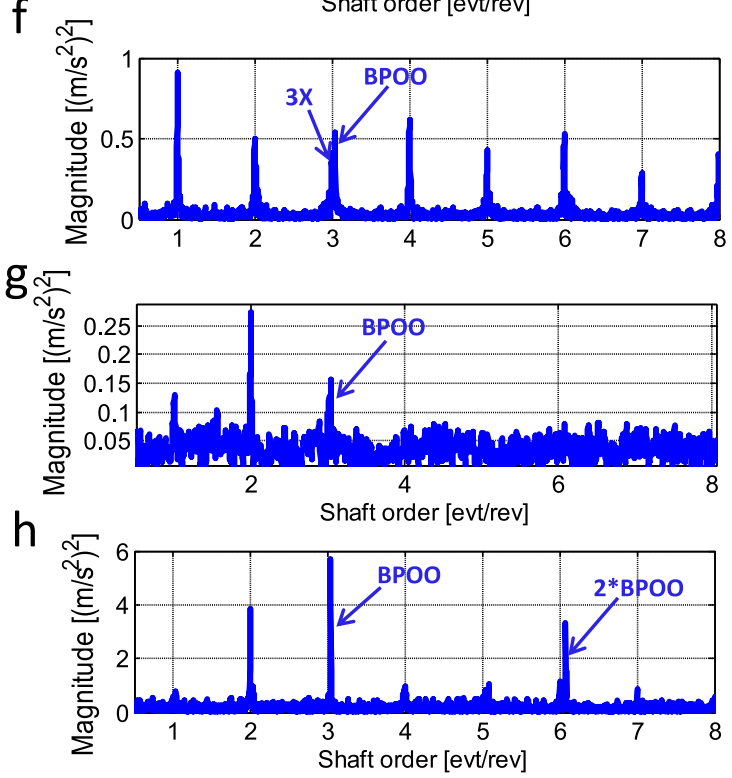

Fig. 16. SES of (a) the resampled signal, (b) the ISA residue, (c) the CPW signal, and (d) the GSA residue in runup case. SES of (e) the resampled signal, ( $\mathrm{f}$ ) the ISA residue, $(\mathrm{g})$ the CPW signal, and $(\mathrm{h})$ the GSA residue in random speed-varying case. Signals acquired by Acc2.

a
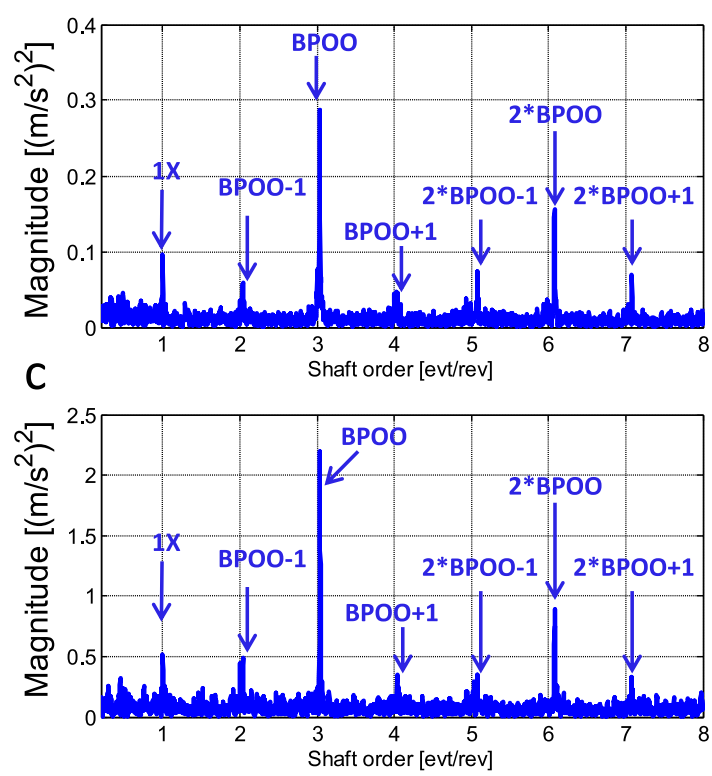

b
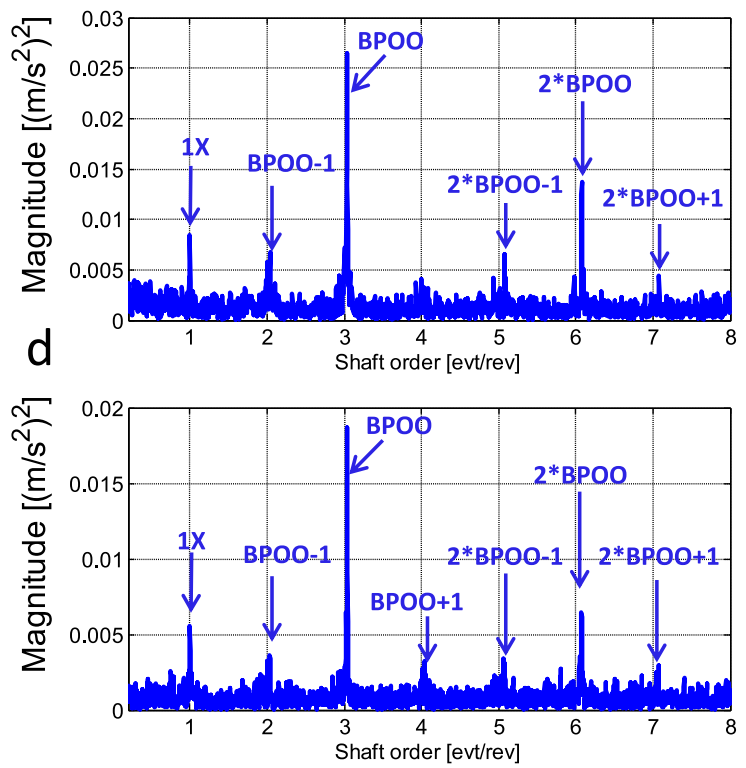

Fig.17. The envelope enhancement methodology applied on the Acc2 signals with filter parameters $\mathbf{f}_{\mathbf{c}}=7 \mathbf{k H z}$ and Bw $=1 \mathbf{k H z}$. Runup case: (a) GSA-based strategy and (b) CPW-based strategy. Random speed-varying case: (c) GSA-based strategy and (d) CPW-based strategy. 


\subsubsection{Enhancing the REB component}

Benefiting from the information on the high-SNR bands of the REB fault (see Table 2), it is useful to enhance the corresponding component by means of the methodology provided in Section 3.2.3. The filter parameters are chosen to account for the largest spectral band - i.e. $\mathrm{f}_{\mathrm{c}}=7 \mathrm{kHz}$ and $\mathrm{Bw}=2 \mathrm{kHz}$. Then, the SES is applied on the order tracked signal and the obtained results are displayed in Fig. 17. Comparing the results to the SE spectra of the whole demodulation band (see Fig. 6(c), (g), (d) and (h)), one can remark improvement at two levels. The first one is the significant decrease in the contribution of the random gear component: the peaks synchronous with the gear rotation are weaker than those related to the REB fault. The second is the complete appearance of a clean outer-race fault signature $m \times \mathrm{BPOO}+\mathrm{n}$ with $m=1,2 ; n= \pm 1$ [11] ${ }^{3}$. The sidebands were completely masked by noise when the SES was applied over the whole demodulation bands. Note also that the filtering operation has decreased the negative effect of the distortion in the CPW case (compare for instance Fig. 16(g) with Fig. 17 (d)).

\subsection{Case study 2: the gear component dominates the random part}

This paragraph treats the case where the accelerometer is mounted on the healthy REB (Acc1). In practice, such cases are likely to be met in complex gearboxes where accelerometers are remoted from the sources due to non-intrusion constraints. This presents a challenging case since Acc1 is also close to the gears; that is the weak fault signature energy is likely to be masked by high-energy cyclic components generated by the gears. In fact, it will be shown that the SES is insufficient to reveal the REB fault signature even after removing the deterministic component by either the GSA or the CPW. This case demonstrates the robustness of the envelope enhancement procedure (provided in Section 3.2.3) in highlighting a particular (very) weak signature surrounded by energetic noises and cyclic components from other sources.

\subsubsection{Failure of the SES}

Hereafter, the SES is applied on Acc1 signal of the two profiles after eliminating the gear component by the GSA and the CPW techniques. The GSA parameters are identical to those chosen in Section 6.2.1. The obtained results are reported in Fig. 18. For the runup profile (see in Fig. 18(a) and (c)), only a small peak at the BPOO appears in the GSA and CPW cases. However, this peak only appears in the GSA case for random-speed varying profile, while it disappears in the CPW cases. Note also that the peaks related to the random gear contribution are also distorted as most of the corresponding peaks disappear except the second harmonic (see Fig. 18(d)). Again, this is due to the high variability of the speed in the record (see Section 5.2.1). This is a case where the SES is insufficient to accurately detect the REB fault. The next subsection addresses this issue by considering the envelope enhancement methodology.

a
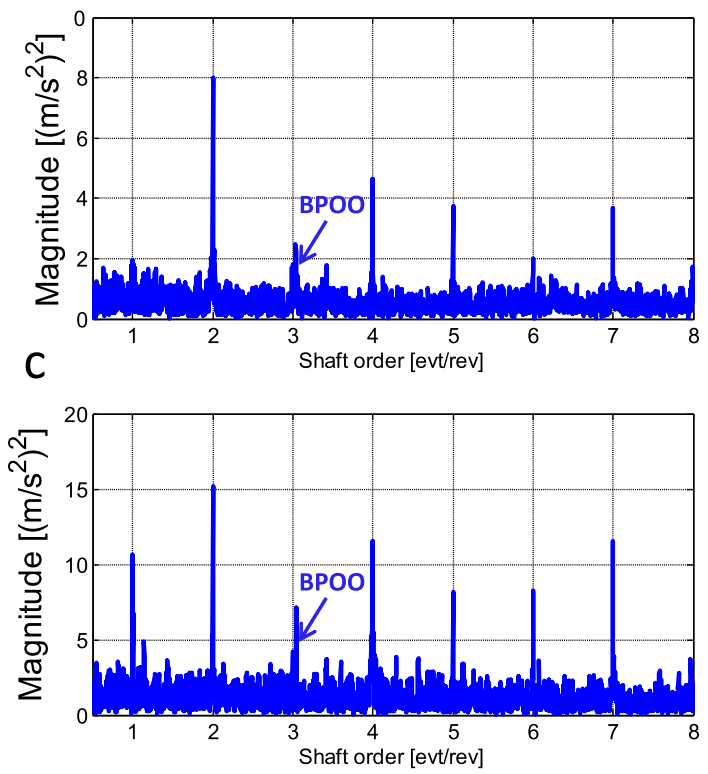

b
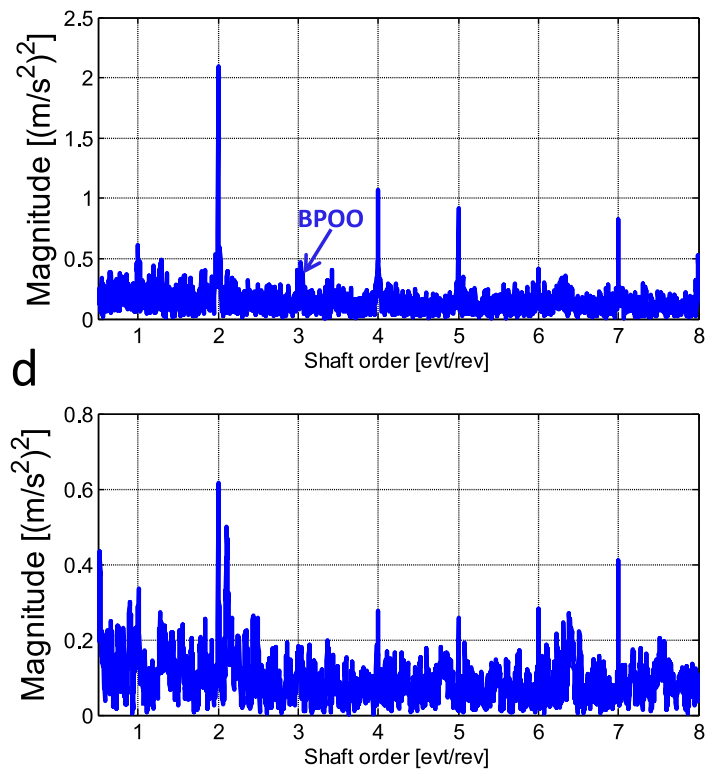

Fig. 18. SES of (a) the GSA residue and (b) the CPW signal in runup case. SES of (c) the GSA residue and (d) the CPW signal in random speed-varying case. Signals acquired by Acc1.

\footnotetext{
${ }^{3}$ The appearance of sidebands in the outer-race fault case may be due to shaft unbalance.
} 
a

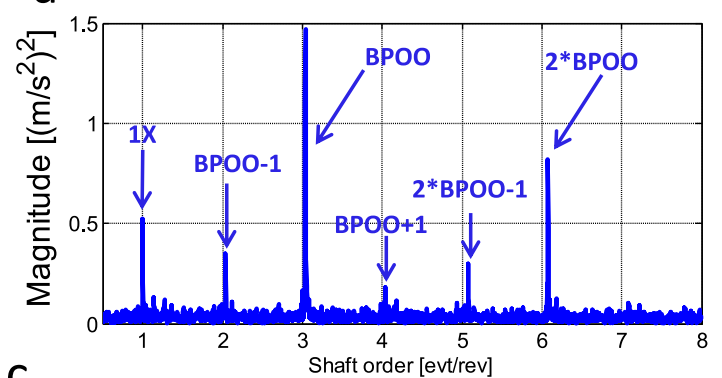

C

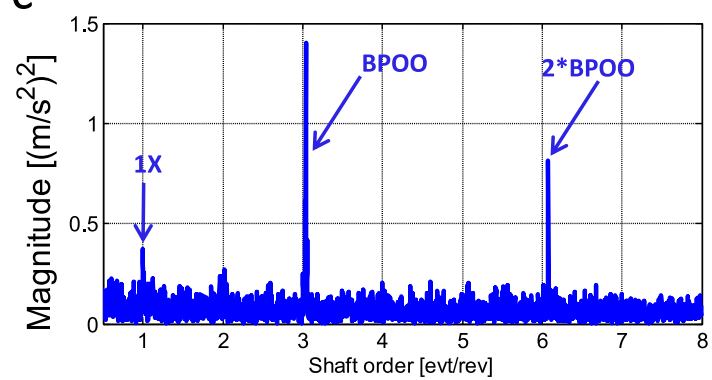

b
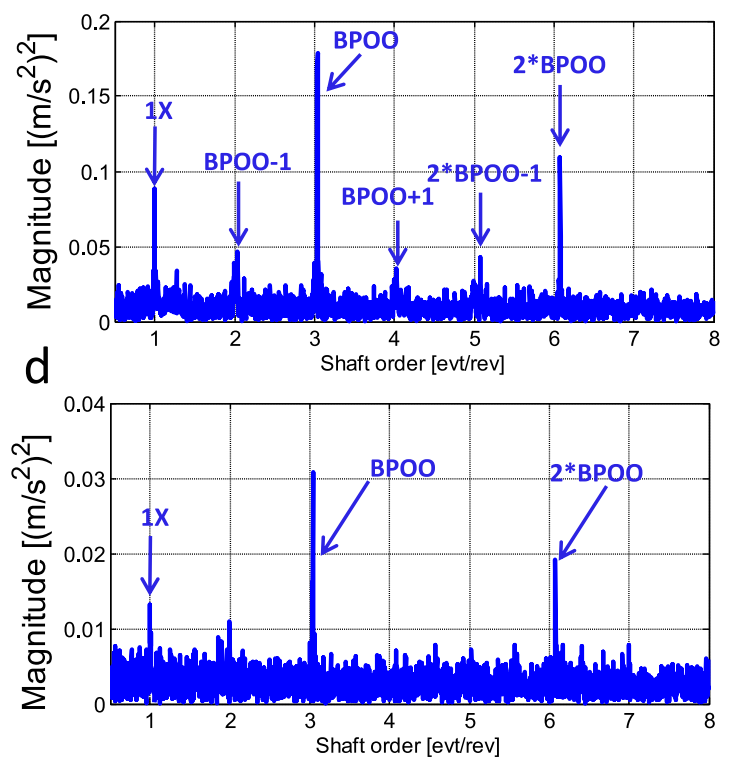

Fig. 19. The envelope enhancement procedure applied on the Acc1 signal with filter parameters $\mathbf{f}_{\mathbf{c}}=7 \mathbf{k H z}$ andBw $=1 \mathbf{k H z}$. Runup case: (a) GSA-based strategy and (b) CPW-based strategy. Random speed-varying case: (c) GSA-based strategy and (d) CPW-based strategy.

\subsubsection{Enhancing the REB component}

In this subsection, the REB component is enhanced using the frequency band identified in Table 2. After eliminating the deterministic component by the GSA or the CPW, the signal is bandpass filtered with the following parameters - i.e. $\mathrm{f}_{\mathrm{c}}=2 \mathrm{kHz}$ and $\mathrm{Bw}=1 \mathrm{kHz}$. Then, the SES is applied on the order tracked signal and the results are displayed in Fig. 19. Interestingly, a clean outer-race fault signature appears for both profiles and strategies. In details, whereas two peaks appear in the SES at the BPOO and its second harmonic in the random speed-varying case (Fig. 19(a) and (b)), sidebands spaced by one order also appear in the runup case (Fig. 19(c) and (d)). Last, it is worth noting that the extracted signature provides an image of the excitation very similar to that obtained for Acc2 (compare Fig. 19 with Fig. 17).

\section{Conclusion}

When dealing with random and repetitive signatures, the investigation of the squared envelope spectrum (SES) is unescapable because of its simplicity, low computational cost and implementation easiness on the one hand and the confidence gained through the decades in industries on the other hand. This paper has revised its application on rotating machine signals in variable speed conditions (VSC). It has collected previously published materials into a comprehensive framework. The aim was to provide guidelines to choose the best strategy for a given benchmark. At the onset, a phenomenological model has been proposed to describe the structure of vibration signals in VSC. This is the cornerstone towards the formalization of the subject for a comprehensive evaluation of existing strategies. Two potential challenges that may face the direct application of the SES have been identified. These are discussed hereafter together with solutions.

The first challenge is the spurious contribution of the deterministic component in the SES. The paper has addressed this issue by comparing three sophisticated methods, namely, the improved synchronous average (ISA), the cepstrum prewhitening (CPW), and the generalized synchronous average (GSA), used for suppressing the deterministic part in a vibration signal. Regarding the ISA, it has been shown that this technique suffers from serious problems as it (i) ignores the long-term energy modulation in the signal and (ii) supposes a linear relationship between phases of the harmonics. Therefore, it returns poor results in the case of modest speed variations and generally fails in large speed variations. Conversely, the CPW technique returns better results for both modest and large speed variations. Often, it suppresses completely the deterministic part, yet suffering also from some disadvantages. The most serious one is the distortion in the random part caused by the whitening operation. Such distortions also increase with the speed variability in the record. The latter case was met in a case-study with large randomly speed-varying profile where the REB fault signature almost disappeared from the envelope. On the other hand, the GSA proves an exceptional ability to systematically discriminate the deterministic part from the random part with minimal distortion of the latter. This technique also has some drawback such as its inability to remove modulation sidebands whose angular period is incommensurate with the carrier period. Note that the ISA shares the latter inconvenient. Building on this, the GSA can be an efficient alternative to the ISA. As for the preference between the GSA and the CPW, it seems strictly dependent on the nature of the application. 
The second challenge is the spurious contribution of CNS components emitted by other sources. This issue has been ignored in the literature where the SES is usually computed over the whole demodulation band after removing the deterministic part. The effectiveness of this approach relies on the prominence of the source of interest (SOI) in the random part. Therefore, it can be efficient for some applications but does not present a versatile solution in the general setting. In this context, it has been shown that the best way to exhibit the mechanical signature a cyclo-non-stationary SOI in the SES is to filter (in the time domain) the signal - hypothetically purely random - around a high-SNR frequency band. In VSC, those bands may be identified, for instance, by an angle/time-cyclostationary (AT-CS) analysis of the emitted signal.

Eventually, the SES itself is an optimal diagnostic indicator in terms of simplicity and computational cost. However, regardless of the adopted strategy, a prior knowledge of high-SNR band associated with the evaluated component is required. This is a strong requirement as it calls for more complex and sophisticated techniques to identify these bands. The straightforward solution to this issue is to scan the entire frequency band. This will bring back to the AT-CS framework through an order-frequency representation. The authors believe that future works are to be oriented toward the exploitation of the SES features in the AT-CS framework.

\section{Acknowledgments}

This work was supported by the technical center of mechanical industries (Cetim). It was performed within the framework of the LabExCeLyA ("Center Lyonnais d'Acoustique", ANR-10-LABX-60).

\section{Appendix A. Proof of equations}

\section{A.1. SES of the deterministic component}

The squared envelope of the deterministic part reads

$$
S E_{d}(t)=\mathbb{E}\left\{|\mathrm{d}(\mathrm{t})|^{2}\right\}=\sum_{\lambda_{j} \in \Lambda} \sum_{\lambda_{k} \in \Lambda} d^{\lambda_{j}}(\omega(t)) d^{\lambda_{k}}(\omega(t))^{*} e^{j\left(\lambda_{j}-\lambda_{k}\right) \theta(t)} .
$$

Applying the change of variable $\lambda_{i}=\lambda_{j}-\lambda_{k}$, one obtains

$$
S E_{d}(t)=\sum_{\lambda_{i} \in \Lambda}\left(\sum_{\lambda_{k} \in \Lambda} d^{\lambda_{i}+\lambda_{k}}(\omega(t)) d^{\lambda_{k}}(\omega(t))^{*}\right) e^{j \lambda_{i} \theta(t)}=\sum_{\lambda_{i} \in \Lambda} S E_{d}^{\lambda_{i}}(\omega(t)) e^{j \lambda_{i} \theta(t)} .
$$

The corresponding SES is obtained by following the same lines as in Eq. (6), i.e.

$$
\operatorname{SES}_{d}(\alpha)=\mathcal{F}_{\theta \rightarrow \alpha}\left\{\operatorname{SE}_{d}(t(\theta))\right\}=\sum_{\lambda_{i} \in \Lambda} \operatorname{SES}_{d}^{\lambda_{i}}\left(\alpha-\lambda_{i}\right),
$$

with $\operatorname{SES}_{d}^{\lambda_{i}}(\alpha)=\mathcal{F}_{\theta \rightarrow \alpha}\left\{S_{d}^{\lambda_{i}}(\tilde{\omega}(\theta))\right\}$ and $\tilde{\omega}(\theta)=\omega(t(\theta))$.

\section{A.2. SES of the CNS component}

Regarding the CNS contribution, the SES is evaluated on the general model provided in Eq. (8). Accordingly, the SE of a CNS signal reads:

$$
S E_{y}(t)=\mathbb{E}\left\{|y(t)|^{2}\right\}=\sum_{\gamma_{j} \in \Gamma_{y}} \sum_{\gamma_{k} \in \Gamma_{y}} \mathbb{E}\left\{y^{\gamma_{j}}(t, \omega(t)) y^{\gamma_{k}}(t, \omega(t))^{*}\right\} e^{j\left(\gamma_{j}-\gamma_{k}\right) \theta(t)} .
$$

Applying the change of variable $\gamma_{i}=\gamma_{j}-\gamma_{k}$, one obtains

$$
S E_{y}(t)=\sum_{\gamma_{i} \in \Gamma_{y}}\left(\sum_{\gamma_{k} \in \Gamma_{y}} \mathbb{E}\left\{y^{\gamma_{i}+\gamma_{k}}(t, \omega(t)) y^{\gamma_{j}}(t, \omega(t))^{*}\right\}\right) e^{j \gamma_{i} \theta(t)}=\sum_{\gamma_{i} \in \Gamma_{y}} S E_{y}^{\gamma_{i}}(\omega(t)) e^{j \gamma_{i} \theta(t)} .
$$

The corresponding SES is obtained by following the same lines as in Eq. (6), i.e.

$$
\operatorname{SES}_{y}(\alpha)=\mathcal{F}_{\theta \rightarrow \alpha}\left\{\operatorname{SE}_{y}(t(\theta))\right\}=\sum_{\gamma_{i} \in \Gamma_{y}} \operatorname{SES}_{y}^{\gamma_{i}}\left(\alpha-\gamma_{i}\right)
$$

with $\Pi_{Y}^{\gamma}(\alpha)=\mathcal{F}_{\theta \rightarrow \alpha}\left\{S E_{y}^{\gamma_{i}}(\tilde{\omega}(\theta))\right\}$. According to this, the SES of the CNS components emitted by the SOI and by the $m$ th interfering source reads 


$$
\left\{\begin{array}{c}
\operatorname{SES}_{S}(\alpha)=\sum_{\gamma_{i}^{(s)} \in \Gamma_{S}} \operatorname{SES}_{S}^{\gamma_{i}^{(s)}}\left(\alpha-\gamma_{i}^{(s)}\right) \\
\operatorname{SES}_{q_{m}}(\alpha)=\sum_{\gamma_{i}^{(m)} \in \Gamma_{m}} \operatorname{SES}_{q_{m}^{\gamma_{i}}}^{\gamma^{(m)}}\left(\alpha-\gamma_{i}^{(m)}\right) .
\end{array}\right.
$$

\section{A.3. SES of the background noise}

Eventually, the SES of the background noise is obtained by simply replacing the cyclic order set $\Gamma_{y}$ in Eq. (A6) by the singleton $\{0\}$, i.e.

$$
\operatorname{SES}_{\mathrm{b}}(\alpha)=\mathcal{F}_{\theta \rightarrow \alpha}\left\{S E_{\mathrm{b}}(t(\theta))\right\}=\operatorname{SES}_{b}^{0}(\alpha)
$$

with $\operatorname{SES}_{b}^{0}(\alpha)=\mathcal{F}_{\theta \rightarrow \alpha}\left\{\operatorname{SE}_{b}^{0}(\tilde{\omega}(\theta))\right\}$ and $\operatorname{SE}_{b}^{0}(\omega)=\mathbb{E}\left\{\left|b^{0}(t, \omega(t))\right|^{2}\right\}$.

Note that the properties of the functions $\operatorname{SES}_{d}^{\lambda_{i}}(\alpha), \operatorname{SES}_{y}^{\gamma_{i}}(\alpha)$ and $\operatorname{SES}_{b}^{0}(\alpha)$ are similar to those of function $D^{\lambda_{i}}(\alpha)$ encountered in Eq. (6).

\section{A.4. Proof of Eq. (30)}

Exploiting the linearity of the GSA:

$$
m_{x}(\bar{\theta}, \omega)=m_{d}(\bar{\theta}, \omega)+m_{r}(\bar{\theta}, \omega) .
$$

Evidently, the GSA of the random part tends to zero as the average number increases toward the infinity. It remains to calculate the GSA on the model of the deterministic part. Inserting Eq. (5) into Eq. (27), one obtains

$$
m_{x}(\bar{\theta}, \omega)=\lim _{\delta \omega \rightarrow 0 \operatorname{card}\left\{K_{\bar{\theta}, \omega}\right\} \rightarrow \infty} \frac{1}{\operatorname{card}\left\{K_{\bar{\theta}, \omega}\right\}} \sum_{k \in K_{\bar{\theta}, \omega}} \sum_{\lambda_{i} \in \Lambda} d^{\lambda_{i}}(\tilde{\omega}(\bar{\theta}+k \Theta)) e^{j \lambda_{i}(\bar{\theta}+k \theta)} .
$$

Similarly to the classical synchronous average, the GSA also needs the cyclic orders to be multiple, i.e. $\lambda_{i}=i . \lambda_{1}=i * 2 \pi / \Theta$ for all $i=2,3 \ldots$. By interchanging the sums, Eq. (A10) becomes

$$
m_{X}(\bar{\theta}, \omega)=\lim _{\delta \omega \rightarrow 0 \operatorname{card}\left\{K_{\bar{\theta}, \omega}\right\} \rightarrow \infty} \sum_{\lambda_{i} \in \Lambda}\left[\frac{1}{\operatorname{card}\left\{K_{\bar{\theta}, \omega}\right\}} \sum_{k \in K_{\bar{\theta}, \omega}} d^{\lambda_{i}}(\tilde{\omega}(\bar{\theta}+k \Theta))\right] e^{j \lambda_{i} \bar{\theta}} .
$$

The rest of the proof follows once it is recognized that $d^{\lambda_{i}}(\tilde{\omega}(\bar{\theta}+k \Theta))=d^{\lambda_{i}}(\omega)$ for all $k \in K_{\bar{\theta}, \omega}$ when $\delta \omega \rightarrow 0$.

\section{A.5. Proof of Eq. (33)}

Exploiting Eq. (9), the filtration of signal, $\tilde{s}(t)$, is expressed as:

$$
\tilde{s}(t)=s(t) \otimes f(t)=\sum_{\gamma_{i}^{(s)} \in \Gamma_{S}} \tilde{s}_{i}^{\gamma_{i}^{(s)}}(t, \omega(t)) e^{j \gamma_{i}^{(s)} \theta(t)}
$$

where $\tilde{s}^{\gamma_{i}}(t, \omega)=s^{\gamma_{i}}(t, \omega) \otimes f(t)$ provided that the impulse response length $f(t)$ is smaller than the cycle duration. Its corresponding SES is

$$
\operatorname{SES}_{\tilde{S}}(\alpha)=\sum_{\gamma_{i}^{(S)} \in \Gamma_{S}} \operatorname{SES}_{\tilde{s}}^{\gamma_{i}^{(s)}}\left(\alpha-\gamma_{i}^{(s)}\right)
$$

where $\operatorname{SES}_{\tilde{S}}^{\gamma_{i}^{(s)}}(\alpha)=\mathcal{F}_{\theta \rightarrow \alpha}\left\{S E_{\tilde{S}}^{\gamma_{i}^{(s)}}(\tilde{\omega}(\theta))\right\}$ and $S E_{\tilde{S}}^{\gamma}(\omega)=\sum_{\gamma^{\prime}} \mathbb{E}\left\{\tilde{s}^{\gamma^{\prime}+\gamma}(\omega) \tilde{S}^{\gamma^{\prime}}(\omega(t))^{*}\right\}$.

\section{Appendix B. Estimation of the GSA}

For estimation purposes, the speed profile is divided into a predefined set of speed intervals called regimes defined by their central frequency $\omega_{r}$ and the speed resolution $\delta \omega$. Then, each cycle is associated to the regime that is closest to its mean speed value. Building on this, the raw estimator is then defined as,

$$
\hat{\mathrm{m}}_{\mathrm{y}}\left(\bar{\theta}, \omega_{\mathrm{r}}\right)=\frac{1}{\operatorname{card}\left\{\mathrm{K}_{\mathrm{r}}\right\}} \sum_{k \in K_{\mathrm{r}}} y(\bar{\theta}+k \Theta)
$$


where $K_{r}=\left\{k \in \mathbb{N}^{*} \omega_{r}-\delta \omega / 2 \leq \frac{1}{\Theta} \int_{(k-1) \Theta}^{k \Theta} \tilde{\omega}(\theta) d \theta<\omega_{r}+\delta \omega / 2\right\}$ stands for the set of cycles whose mean speed falls in the speed interval centered at $\omega_{r}$. Note that the raw estimate of the GSA provides only estimates at the central frequencies of the predefined speed intervals. Thus, the deterministic component could not be accurately restored by Eq. (28). For this reason, an interpolation is needed to enforce the continuity along the speed axis. By applying the kernel density estimation technique, an estimation of the (synchronous) deterministic part is provided by

$$
\hat{m}_{y}(\bar{\theta}, \omega)=\frac{1}{\operatorname{Kern}\left(\frac{\tilde{\omega}(\theta)-\omega_{r}}{\sigma}\right)} \sum_{r=1}^{R} \operatorname{Kern}\left(\frac{\tilde{\omega}(\theta)-\omega_{r}}{\sigma}\right) \hat{m}_{y}\left(\bar{\theta}, \omega_{r}\right)
$$

where $\operatorname{Kern}(*)$ is the smoothing kernel (e.g. Gaussian, Hamming...), $\sigma$ is a smoothing parameter called the bandwidth, $\hat{m}_{y}\left(\theta, \omega_{r}\right)$ is the periodized version of $\hat{m}_{y}\left(\bar{\theta}, \omega_{r}\right)$ over the actual number of periods of $y(\theta)$.

\section{Appendix C. Simulation models for gears and bearings}

\section{C.1. Deterministic contribution of gears}

The deterministic gear contribution is principally induced by the meshing force and the fault impacts. The former manifests itself as a smooth angle-periodic component of fundamental order equal to the number of teeth $Z_{1}$ while the latter manifests itself as an impulsive angle-periodic component of fundamental order equal to the wheel rotation, i.e. 1. The interaction between these excitations is mostly manifested through an amplitude modulation. In turn, the resulting excitation undergoes speed-dependent energy modulation caused by the change of the machine power intake as the speed varies. Building on this, one can model the excitation of the deterministic gear vibration part as

$$
\Sigma_{g}^{(d)}(t)=M_{g}^{(d)}\left(\omega_{r e f}(t)\right)\left(1+I\left(\theta_{\text {ref }}(t)\right)\right) \sum_{l} a_{l} \cos \left(Z_{1} \cdot l \cdot \theta_{\text {ref }}(t)+\varphi_{l}\right),
$$

where $\theta_{\text {ref }}(t)=\int_{0}^{t} \omega_{\text {ref }}(t) d t$ stands of the angular position of the reference shaft rotating with the angular frequency $\omega_{\text {ref }}$, $M_{g}^{(d)}\left(\omega_{\text {ref }}\right)$ is a monotonally increasing modulation function with respect to the speed, $a_{l}$ and $\varphi_{l}$ are the $l$ th Fourier coefficients of the angle-periodic meshing excitation, and

$$
I\left(\theta_{\text {ref }}\right)=\sum_{i} \mathrm{I}_{\xi}\left(\theta_{\text {ref }}-\mathrm{i} .2 \pi\right)
$$

models the fault impacts, where $\mathrm{I}_{\xi}\left(\theta_{\text {ref }}\right)$ stands for a Hanning window of length, $\xi$, representing the angular duration of the impact. This latter is supposed to be constant in the angular domain as it may actually depend on the fault width. The deterministic gear vibration signal is then obtained after passing through an LTI system, say $h_{g}^{(r)}(t)$, that physically expresses the transmission path from the excitation to the sensor:

$$
d(t)=\Sigma_{g}^{(d)}(t) \otimes h_{g}^{(d)}(t)
$$

\section{C.2. Random contribution of gears}

The random contribution of the output gear can be modeled as the product of white noise modulated by an angleperiodic function with period equal to that of the output gear. By adding the speed-dependent energy modulation caused by the change of the machine power intake, one can model the random contribution of the output gear as:

$$
q(t)=M_{g}^{(r)}\left(\omega_{r e f}(t)\right) W(t) \sum_{m} a_{m} \cos \left(\frac{Z_{1}}{Z_{2}} \cdot m \cdot \theta_{r e f}(t)+\varphi_{m}\right)
$$

where $M_{g}^{(r)}\left(\omega_{\text {ref }}\right)$ is a monotonally increasing modulation function with respect to the speed, $W(t)$ is a white noise of unit standard deviation, $a_{m}$ and $\varphi_{m}$ are the $m$ th Fourier coefficients of the angle-periodic meshing excitation. The random gear signal is then obtained after passing through an LTI system, say $h_{g}^{(r)}(t)$, that physically expresses the transmission path from the excitation to the accelerometer:

$$
X_{g}^{(r)}(t)=\Sigma_{g}^{(r)}(t) \otimes h_{g}^{(r)}(t)
$$

\section{C.3. Random contribution of REB}

In the following, a cyclostationary-based bearing vibration model introduced in [11] is revisited to account for speed 
variation. The presence of a local fault in a REB component produces a train of short time impulses, angle periodic on the average, locked to the fault characteristic angular period. The inter-arrival angles of the impacts are subjected to slight random fluctuations due to existing slips, whereas the corresponding magnitude is subject to fluctuations due to the nonexactly reproducible microscopic conditions when a rolling surface impacts the fault. Similarly to the gear case, the excitation is also subjected to speed dependent energy changes. In this model, the modulation effects caused by the passage in the load zones are neglected. A faulty rolling element bearing signal is then obtained by exciting a wide band structural resonance as,

$$
s(t)=M_{b}\left(\omega_{r e f}(t)\right) \sum_{i} A_{i} h_{b}\left(t-T_{i}\right), \text { with } T_{i}=t\left(i . \Theta_{f}-\mu_{i}\right)
$$

where $M_{b}\left(\omega_{\text {ref }}\right)$ is a monotonally increasing modulation function, $h_{b}$ is the system impulse response, $\Theta_{f}=2 \pi / B P O O$ is the fault characteristic angular period, $A_{i}$ are the amplitude modulations modeled as mutually delta-correlated random sequence with $\mathbb{E}\left\{A_{i}\right\}=\bar{A}$ and $\operatorname{cov}\left\{A_{i}, A_{j}\right\}=\sigma_{A}^{2} \delta_{i j}, \mu_{i}$ are the inter-arrival angle fluctuations modeled as mutually delta-correlated random variable with $\mathbb{E}\left\{\mu_{i}\right\}=0$ and $\operatorname{cov}\left\{\mu_{i}, \mu_{j}\right\}=\sigma_{\mu}^{2} \delta_{i j}$.

\section{C.4. Background noise}

Eventually, background noise $b(t)$ is modeled by a stationary white Gaussian noise modulated by a speed-dependent monotonically increasing function $M_{N}\left(\omega_{r e f}\right)$,

$$
b(t)=W(t) M_{N}\left(\omega_{r e f}(t)\right)
$$

Finally, the complete vibration signal can be then obtained by summing all contributions,

$$
x(t)=\mathrm{d}(t)+\mathrm{s}(t)+q(t)+b(t)
$$

For simplicity, the LTI filter $h_{g}^{(d)}(t), h_{g}^{(d)}(t)$ and $h_{b}(t)$ are chosen to be the impulse responses of single-degree-of-freedom (s. d.o.f.) systems with different parameters.

\section{References}

[1] M.S. Darlow, R.H. Badgley, G.W. Hogg, Application of high frequency resonance techniques for bearing diagnostics in helicopter gearboxes, Technical Report, US Army Air Mobility Research and Development Laboratory, 1974, pp. 74-77.

[2] D. Ho, R.B. Randall, Optimisation of bearing diagnostic techniques using simulated and actual bearing fault signals, Mech. Syst. Signal Process. 14 (5) (2000) 763-788.

[3] R.B. Randall, Hilbert transform techniques in machine diagnostics, in: Proceedings of IFToMM International Conference on Rotordynamics, Tokyon, 1986.

[4] J. Urbanek, J. Antoni, T. Barszcz, Detection of signal component modulations using modulation intensity distribution, Mech. Syst. Signal Process. 28 (2012) 399-413.

[5] A. Jabłoński, J. Urbanek, T. Barszcz, Application of angular-temporal spectrum for detection of rolling-element bearing faults operating under varying speed regime, in: Proceedings of the 4th Conference in Condition Monitoring of Machinery in Non-Stationary Operations, Lyon, France, 14-16 December, 2014.

[7] K.R. Fyfe, E.D.S. Munck, Analysis of computed order tracking, Mech. Syst. Signal Process. 11 (2) (1997) 187-205.

[8] J. Antoni, Cyclostationary by examples, Mech. Syst. Signal Process. 23 (4) (2009) 987-1036.

[9] J. Antoni, Cyclic spectral analysis in practice, Mech. Syst. Signal Process. 21 (2007) 597-630.

[10] J. Antoni, F. Bonnardot, A. Raad, M. El Badaoui, Cyclostationary modelling of rotating machine vibration signals, Mech. Syst. Signal Process. 18 (2004) $1285-1314$.

[11] R.B. Randall, J. Antoni, S. Chobsaard, The relationship between spectral correlation and envelope analysis for cyclostationary machine signals: application to ball bearing diagnostics, Mech. Syst. Signal Process. 15 (5) (2001) 945-962.

[12] S. Braun, B. Seth, Analysis of repetitive mechanism signatures, J. Sound Vib. 70 (4) (1980) 513-522.

[13] G. Lejeune, J.L. Lacoume, P. Marchand, M. Durnerin, N. Martin, J. Liénard, A. Silvent CEPHAG, Cyclostationnarité d'ordre 1 et 2: application à des signaux vibratoires d'engrenages, 16 i'eme colloque GRETSI, Grenoble, September 1997, pp. 323-326 (in French).

[14] J. Antoni, Apport de l'échantillonnage angulaire et de la cyclostationarite au diagnostic par analyse vibratoire des moteurs thermiques (Ph.D. dissertation), 2003 (in French).

[15] J. Antoni, M. Eltabach, A KIS solution for high fidelity interpolation and resampling of signals, Mech. Syst. Signal Process. 35 (1-2) (2013) 127-136.

[16] D. Abboud, S. Baudin, J. Antoni, D. Remond, M. Eltabach, O. sauvage, The spectral correlation analysis of cyclo-non-stationary signals, Mech. Syst. Signal Process. 75 (2016) 280-300.

[17] J. Antoni, Cyclic spectral analysis of rolling-element bearing signals: facts and fictions, J. Sound Vib. 304 (2007) 497-529.

[18] J. Antoni, Cyclostationarity by examples, Mech. Syst. Signal Process. 23 (2009) 987-1036.

[19] R.B. Randall, J. Antoni, Rolling element bearing diagnostics - a tutorial, Mech. Syst. Signal Process. 25 (2) (2011) 485-520.

[20] R.B. Randall, Vibration-Based Condition Monitoring: Industrial, Automotive and Aerospace Applications, John Wiley and Sons, West Sussex, 2011.

[21] M.D. Coats, N. Sawalhi, R.B. Randall, Extraction of tacho information from a vibration signal for improved synchronous averaging, Proc. Acoust. (2009).

[22] C.J. Stander, P.S. Heyns, Transmission path phase compensation for gear monitoring under fluctuating load conditions, Mech. Syst. Signal Process. 20 (7) (2006) 1511-1522.

[23] P. Borghesani, P. Pennachi, R.B. Randall, R. Ricci, Order tracking for discrete-random separation in variable speed conditions, Mech. Syst. Signal Process. 30 (2012) 1-22.

[24] N. Sawalhi, R.B. Randall, Signal pre-whitening using cepstrum editing (Liftering) to enhance fault detection in rolling element bearing, in: Proceedings of the 24th international Congress in Condition Monitoring and Diagnostics Engineering Management (COMADEM), 2011.

[25] P. Borghesani, P. Pennacchi, R.B. Randall, N. Sawalhi, R. Ricci, Application of cepstrum pre-whitening for the diagnosis of bearing faults under variable 
speed conditions, Mech. Syst. Signal Process. 36 (2013) 370-384.

[26] D. Abboud, J. Antoni, S. Sieg-Zieba, M. Eltabach, Deterministic-random separation in non-stationary conditions, J. Sound Vib. 362 (2016) 305-326.

[27] D. Abboud, J. Antoni, S. Sieg-Zieba, M. Eltabach, Angle/time cyclostationarity for the analysis of rolling element bearing vibrations, Measurements 75 (2015) 29-39.

[28] P. Borghesani, R. Ricci, S. Chatterton, P. Pennacchi, A new procedure for using envelope analysis for rolling element bearing diagnostics in variable operating conditions, Mech. Syst. Signal Process. 36 (2013) 370-384.

[29] J. Antoni, D. Abboud, S. Baudin, Time-angle periodically correlated process, Cyclostationarity: theory and methods, Lecture Notes in Mechanical Engineering, Springer, 2014.

[30] J. Antoni, Cyclic spectral analysis in practice, Mech. Syst. Signal Process. 21 (2007) 597-630.

[31] J. Antoni, J. Schoukens, A comprehensive study of the bias and variance of frequency-response-function measurements: optimal window selection and overlapping strategies, Automatica 43 (10) (2007) 1723-1736.

[32] S. Baudin, D. Rémond, J. Antoni, O. Sauvage, Detection of Rattle Noise with Optical Encoders in Run-up Conditions, ISMA, Belgium (2014), p. , 2014, 254.

[33] G. D'Elia, Z. Daher, J. Antoni, A novel approach for the cyclo-nonstationary analysis of speed varying signals, in: Proceedings of ISMA 2010 Including USD, 2010

[34] J. Roussel, M. Haritopoulos, E. Sekko, C. Capdessus, J. Antoni, Application of speed transform to the diagnosis of a roller bearing in variable speed, in: Proceeding of the Conference Surveillance 7, France, October 29-30, 2013.

[35] J. Urbanek, T. Barszcz, J. Antoni, Time-frequency approach to extraction of selected second-order cyclostationary vibration components for varying operational conditions, Measurement 46 (2013) 1454-1463.

[36] M. Inalpolat, A Theoretical and experimental investigation of modulation sidebands of planetary gear sets (Dissertation Ph.D. thesis), Ohio State University, Ohio, 2009.

[37] M. Zhao, J. Lin, X. Xu, Y. Lei, Tacholess envelope order analysis and its application to fault detection of rolling element bearings with varying speeds, Sensors 13 (8) (2013) 10856-10875.

[38] M. Cocconcelli, L. Bassi, C. Secchi, C. Fantuzzi, R. Rubini, An algorithm to diagnose ball bearing faults in servomotors running arbitrary motion profiles, Mech. Syst. Signal Process. 27 (2012) 667-682.

[39] W. Gardner, Introduction to Random Processes, second ed. McGraw-Hill, New York, 1990.

[40] P. Prieur, G. D'urso, Des indices de cyclostationnarité pour la surveillance des engrenages, 15ième Colloque Gretsi, Juan les Pins, 1995, pp. 1241-1244 (in French).

[42] R. Rubini, M. Sidahmed, Diagnostics of Gear Systems Using the Spectral Correlation Density of the Vibration Signal, IFAC Fault Detection, Supervision and Safety for Technical Processes, Kingston Upon Hull, UK, 1997.

[43] C. Capdessus, M. Sidahmed, J.L. Lacoume, Cyclostationary processes: application in gear faults early diagnosis, Mech. Syst. Signal Process. 14 (3) (2000) 371-385.

[44] L. Bouillaut, M. Sidahmed, Cyclostationary approach and bilinear approach: comparison, applications to early diagnosis for helicopter gearbox and classification method based on HOCS, Mech. Syst. Signal Process. 15 (5) (2000) 923-943.

[45] I. Antoniadis, G. Glossiotis, Cyclostationary analysis of rolling-element bearing vibration signals, J. Sound Vib. 248 (5) (2001) $829-845$.

[46] D. Konig, F. Bohme, Application of cyclostationary and time-frequency analysis to car engine diagnosis, IEEE Int. Conf. Acoust. Speech Signal Process. 4 (1994) 149-152.

[47] D. Konig, C. Tork, J. Bohme, Design of optimum periodic time varying filters for applications in combustion diagnosis of car engines, Int. Conf. Acoust. Speech Signal Process. 3 (1995) 1924-1927.

[48] J. Antoni, J. Daniere, F. Guillet, Effective vibration analysis of IC engines using cyclostationarity. Part I: a methodology for condition monitoring, J. Sound Vib. 257 (5) (2002) 815-837.

[49] W. Bartelmus, R. Zimroz, A new feature for monitoring the condition of gearboxes in non-stationary operating conditions, Mech. Syst. Signal Process. 23 (5) (2009) 1528-1534.

[50] W. Bartelmus, F. Chaari, R. Zimroz, M. Haddarb, Modelling of gearbox dynamics under time-varying nonstationary load for distributed fault detection and diagnosis, Eur. J. Mech. - A/Solids 29 (4) (2010) 637-646.

[51] L.F. Villa, A. Renones, J.R. Peran, L.J. de Miguel, Statistical fault diagnosis based on vibration analysis for gear test-bench under non-stationary conditions of speed and load, Mech. Syst. Signal Process. 29 (2012) 436-446.

[52] T. Heyns, S.J. Godsill, J.P. de Villiers, P.S. Heyns, Statistical gear health analysis which is robust to fluctuating loads and operating speeds, Mech. Syst. Signal Process. 27 (2012) 651-666.

[53] S. Baudin, D. Rémond, J. Antoni, Olivier Sauvage, Detection of Rattle Noise with Optical Encoders in Run-up Conditions, ISMA, Belgium (2014), p. , 2014, 254.

[54] S. Baudin, J. Antoni, D. Rémond, O. Sauvage, Détection du bruit de grenaille par analyse cyclo-non-stationnaire, in: Proceedings of CFA, 2014 (in French).

[55] D. Abboud, J. Antoni, S. Sieg-Zieba, M. Eltabach, Generalization of the synchronous average for deterministic/random separation under speed varying conditions, in: Proceedings of VISHNO, 2014.

[56] D. Abboud, J. Antoni, S. Sieg-Zieba, M. Eltabach, Speed-spectral whitening for enhancing envelope analysis in speed varying conditions, in: Proceedings of Vibrations Shocks and Noise, 17-19 June 2014, Aix-en-Provence, France.

[57] D. Abboud, J. Antoni, S. Sieg-Zieba, M. Eltabach. On the extraction of rolling-element bearing fault signature in speed-varying conditions, in: Proceedings of the Eleventh International Conference on Condition Monitoring and Machinery Failure Prevention Technologies, 10-12 June 2014, Manchester, UK.

[58] M.D. Coats, R.B. Randall, Single and multi-stage phase demodulation based order-tracking, Mech. Syst. Signal Process. 44 (2014) $86-117$.

[59] C. Harris, A. Persol, Harris' Shock and Vibration Handbook, McGraw Hill, New York, 2001.

[60] F. Rieger Neville, The Relation Between Finite Element Analysis and Modal Analysis, Stress Technology Incorporated, Rochester, New York, 2008.

[61] J. Antoni, R.B. Randall, The spectral kurtosis: application to the vibratory surveillance and diagnostics of rotating machines, Mech. Syst. Signal Process 20 (2006) 308-331.

[62] J. Antoni, Fast computation of the kurtogram for the detection of transient faults, Mech. Syst. Signal Process. 21 (2007) $108-124$.

[63] T. Barszcz, A. Jablonski, A novel method for the optimal band selection for vibration signal demodulation and comparison with the Kurtogram, Mech. Syst. Signal Process. 25 (2011) 431-451.

[64] P. Borghesani, M.R. Shahriar, Cyclostationary analysis with logarithmic variance stabilisation, Mech. Syst. Signal Process. 70 (2016) 51-72. 2016.

[65] P. Borghesani, P. Pennacchi, R. Ricci, S. Chatterton, Testing second order cyclostationarity in the squared envelope spectrum of non-white vibration signals, Mech. Syst. Signal Process. 40 (1) (2013) 38-55.

[66] Z. Daher, E. Sekko, J. Antoni, C. Capdessus, L. Allam, Estimation of the synchronous average under varying rotating speed condition for vibration monitoring, in: Proceedings of ISMA, 2010. 